\title{
NUMERICAL STUDY OF MAGNETOHYDRODYNAMIC NATURAL CONVECTION IN A NON-DARCIAN POROUS ENCLOSURE FILLED WITH ELECTRICALLY CONDUCTING HELIUM GAS
}

\author{
O. Anwar Bég ${ }^{A}$, K. Venkatadri ${ }^{B}$, V.R. Prasad ${ }^{\text {C* }}$, T. A. Bég ${ }^{D}$, Henry J. Leonard ${ }^{A}$, R.S.R. Gorla ${ }^{\text {E }}$, P. Rajarajeswari $^{\mathrm{F}}$

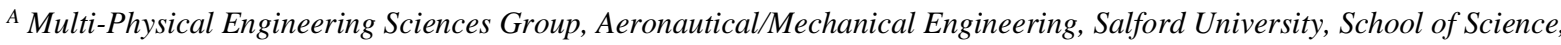 \\ Engineering and Environment (SEE), Manchester, M54WT, UK. \\ Email: O.A.Beg@salford.ac.uk \\ h.leonard@salford.ac.uk \\ ${ }^{B}$ Dept. Mathematics, Sreenivasa Institute of Technology/Management Studies, Chittoor, Andhra Pradesh, 517127, India. \\ Email: venkatadri.venki@gmail.com \\ Dept. Mathematics, School of Advanced Sciences, Vellore Institute of Technology, Vellore - 6322014, India. \\ Email: rcpmaths@gmail.com \\ ${ }^{D}$ Engineering Mechanics Research, Israfil House, Dickenson Rd., Manchester, M13, UK. \\ tasveerabeg@gmail.com \\ ${ }^{E}$ Department of Aeronautics and Astronautics, Airforce Institute of Technology, Dayton, Ohio, USA. \\ Email: rama.gorla@yahoo.com \\ ${ }^{F}$ Department of Computer Science and Engineering, Kingston Engineering College, Vellore-632059, India \\ Email:rajacse77@gmail.com
}

*Corresponding author: Email: $\underline{\text { rcpmaths@gmail.com }}$

\begin{abstract}
A theoretical and computational study of MHD natural convection in an isotropic non-Darcian porous medium saturated with electrically conducting helium gas in an enclosure in the presence of heat generation is presented. A Brinkman extended Darcy-Forchheimer model is employed and the working fluid is assumed to be incompressible. The model is nondimensionalised and converted into pressure-velocity form. The Harlow-Welch marker and cell (MAC) finite difference technique is employed to solve the nonlinear boundary value problem via pressure-vorticity coupling. A parametric investigation of the influence of Grashof number $(G r)$, Hartmann magnetic number $(H a)$, Darcy number $(D a)$, and the internal heat generation parameter $(\Gamma)$ on streamline and isotherm distributions with Prandtl number $(\operatorname{Pr})$ is
\end{abstract}


0.71 (Helium) is conducted. The variation in local Nusselt number along the left and right walls of the computational 2D enclosure is also studied. Validation house-computational numerical MATLAB code is tests are included. Local Nusselt number is elevated at both left and right walls with greater Darcy number (higher medium permeability) and Grashof number. However, with greater internal heat generation, local Nusselt number magnitudes are enhanced at the left (cold) wall only but suppressed at the right (hot) wall. Increasing magnetic field reduces local Nusselt number at both left and right walls. With increasing magnetic field, the single vortex is strongly distorted and skewed towards the top left and lower right corners of the enclosure. Temperature contours at the left and right wall are however less intense with greater magnetic field effect. The simulations are of relevance to hybrid electromagnetic gaseous fuel cells, magnetic field control of filtration processes and porous media materials processing systems.

KEY WORDS: Magnetohydrodynamics, Helium, Internal heating; non-Darcy porous media; finite difference solution; nonlinear convection; Grashof number; hybrid fuel cells.

\section{NOMENCLATURE}

A aspect ratio $(\mathrm{L} / \mathrm{H})$

B uniform magnetic field vector (Tesla)

$B_{o} \quad$ magnitude of $\boldsymbol{B}$ (Tesla)

$C_{p} \quad$ isobaric specific heat $(\mathrm{J} / \mathrm{kgK})$

Da Darcy number

$g \quad$ acceleration due to gravity $\left(\mathrm{m} / \mathrm{s}^{2}\right)$ 
Gr Grashof number

$H \quad$ height of enclosure (m)

Ha Hartmann hydromagnetic number

J electrical current density (Amperes $\left./ \mathrm{m}^{2}\right)$

$K \quad$ permeability of the porous medium $\left(\mathrm{m}^{2}\right)$

L length of enclosure $(m)$

$\mathrm{Nu} \quad$ Nusselt number

p hydrodynamic pressure $(\mathrm{Pa})$

Pr Prandtl number

$q \quad$ heat flux $\left(w / m^{2}\right)$

$q$,", volumetric internal heat generation rate $\left(\mathrm{W} / \mathrm{m}^{3}\right)$

$\mathrm{Ra} \quad$ Rayleigh number

RaI Internal Rayleigh number

$t \quad$ time $(s)$

T temperature $(K)$

$u \quad x$-directionvelocity $(\mathrm{m} / \mathrm{s})$

U dimensionless $u$ velocity

$\boldsymbol{V}$ field velocity vector $(\mathrm{m} / \mathrm{s})$ 
$v \quad y$-directionvelocity $(\mathrm{m} / \mathrm{s})$

$V \quad$ dimensionless $v$ velocity

$x \quad$ co-ordinate parallel to base of enclosure $(m)$

$X \quad$ dimensionless $x$ co-ordinate

y co-ordinate perpendicular to base of enclosure $(m)$

Y dimensionless y co-ordinate

\section{Greek symbols}

$\tau \quad$ dimensionless time

$\sigma \quad$ electrical conductivity of fluid (Siemens/m)

$\beta \quad$ thermal expansion coefficient of fluid $(/ K)$

$\varphi \quad$ electric potential (Volts)

$\mu \quad$ dynamic viscosity of fluid $(\mathrm{kg} / \mathrm{ms})$

$v \quad$ kinematic viscosity of fluid $\left(\mathrm{m}^{2} / \mathrm{s}\right)$

$\rho \quad$ fluid density at reference temperature $\left(\mathrm{kg} / \mathrm{m}^{3}\right)$

$\alpha \quad$ thermal diffusivity of fluid-saturated porous medium $\left(\left(\mathrm{m}^{2} / \mathrm{s}\right)\right.$

$\theta \quad$ dimensionless temperature

$\psi \quad$ stream function 


\section{Subscripts and Superscripts}

( ) Hot wall (right of enclosure)

( ) Cold wall (left of enclosure)

\section{INTRODUCTION}

Transport in porous media continues to find extensive applications in medicine, energy systems, chemical process engineering and geosciences. These studies include both fluid flow and also convective heat and mass transfer in permeable materials arise in for example solar absorber collector systems [1], foam processing [2], geothermal power [3], borehole heat exchangers [4], wavy surface hybrid heat transfer devices [5, 6], biomechanics [7], gastric biotransport [8], bio-fuel cells [9] and industrial filtration [10]. In a number of technologies, flow and heat transfer in enclosure (cavities) are of particular interest including modern fuel cell technologies and materials fabrication (crustal growth etc). Engineers have therefore examined extensively natural convection flows in enclosures filled with porous media, both experimentally and also with a variety of modelling approaches. Both isotropic and anisotropic media have been analysed by a variety of researchers. Martins-Costa et al. [11] applied a continuous theory of mixtures and a control volume numerical method to compute the natural convection flow in a two-dimensional fluid-saturated porous cavity. Saleh and Hashim [12] used a finite difference technique and Darcy model to simulate the impact of a conductive boundary on free convection flow in a square porous enclosure with internal heating proportional to a power of temperature difference. They observed that at very low Rayleigh number (buoyancy parameter), the heat transfer across the cavity remain stable for any values of the thermal conductivity ratio and also that temperature is enhanced more markedly with 
substantial internal heating than via a conductive solid wall. Increasing value thermal conductivity ratio and/or decreasing the thickness of solid wall can increase the maximum fluid temperature. Siddheshwar and Siddabasappa [13] investigated the thermoconvective stability in a fluid-saturated sparsely packed porous medium with local-thermal-non-equilibrium (LTNE) effect. They deployed free-free, adiabatic and rigid-rigid, adiabatic boundary conditions for the vertical walls and stress-free, isothermal and rigid-rigid, isothermal boundary combinations for the horizontal walls. They showed that the onset of convection is faster in a shallow enclosure followed by that in square and tall enclosures. They also computed the influence of Brinkman number and the inter-phase heat transfer coefficient on onset of convection showing that these parameters delay it. Basak et al. [14] utilized a Darcy model and finite element technique to simulate the impact of uniform and non-uniform heating of the base wall on natural convection flows within isosceles triangular enclosures filled with a porous medium. They showed that at small Darcy number (dimensionless permeability parameter), the heat transfer is primarily due to conduction at any value of Prandtl number, whereas with higher Darcy number convection is found to be the dominant heat transfer mechanism and circulation cell structures are more strongly influenced. They also observed that average Nusselt number for the base wall is double that of either inclined wall. Chen et al. [15] computed the natural convection in a square porous cavity with two different local thermal models (local thermal equilibrium model and the local thermal non-equilibrium model) using a spectral method with Chebyshev-Gauss-Lobatto collocation points. They presented detailed simulations for streamlines and isotherms for various Rayleigh number, inter-phase heat transfer coefficient, and thermal conductivity ratio values. Song and Viskanta [16] simulated the free convection flow in a rectangular enclosure partially filled with an anisotropic porous medium as a model of the mushy region flow characteristics on the interacting flows of the melt pool and mushy zone in alloy solidification. They employed volume-averaged conservation equations and a 
Darcy formulation. Further studies include Bég et al. [17] who used MAPLE quadrature and variational finite element methods to compute the swirling heat transfer in natural convection from a rotating conical body to a high permeability anisotropic porous medium. They showed that the tangential flow is accelerated whereas the swirl flow and temperature are reduced with increasing swirl Darcy number and tangential Darcy number.

Since porous media are generally heterogenous in nature and feature randomly distributed pore spaces, simpler approaches have been deployed to simulate transport through such media. These include the Darcy model (refs [11]-[17]) which applies to steady flow through porous media and assumes the flow rate is proportional to the applied pressure gradient. However, when the porosity of the porous medium is close to unity and the regime is highly permeable, the flow of fluid is curvilinear, and curvature of the path gives rise to an inertia effect. As the inertia force increases relative to the viscous force, the streamlines become more distorted and drag increases more rapidly with velocity. An inertial drag term is therefore required and the Darcy-Forchheimer model is often used. As the velocity increases, Reynolds number is within the range $1-10$; however, this transition is not from laminar to turbulent. However, in this regime of Reynolds number, a change from linear drag to non-linear drag is observed. If quadratic drag is incorporated it leads to a decelerate in flow in the porous regime [18]. The boundary layers around the pores become more pronounced and an "inertial core" appears with higher Forchheimer (nonlinear) drag effect. The developing of these "core" flows external to the boundary layers accounts for the non-linear relationship between pressure drop and flow rate. A number of Darcy-Forchheimer studies have been reported in recent years in a large spectrum of applications in porous media transport including heat transfer. Bég et al. [19] computed the two-phase hemodynamic flow and convection heat transfer in a vertical channel 
containing a Darcy-Forchheimer porous medium with the Zhou differential transform method (DTM). They showed that Darcy drag force which arises from the viscous contribution to stress at the solid particle boundaries, exerts progressively less resistance with increasing permeability (i.e. Darcy number) owing to a depletion in porous fibers. They also confirmed that inertial effects due to the porous medium are experienced via the quadratic drag term i.e. Forchheimer term which does not explicitly involve viscosity but does arise from viscosity action, mediated by the inertial effects affecting the distribution of pressure which also contributes to the stress at the solid boundary i.e. channel wall. They further showed that Nusselt numbers at the channel wall are considerably modified with both Darcian and Forchheimer drag force terms. Chandra and Satyamurty [20] simulated the natural convective flow in an anisotropic fluid filled porous rectangular enclosure with end-to-end temperature difference using a Brinkman extended non-Darcy flow model and successive accelerated replacement (SAR) scheme. They observed that average Nusselt number is elevated with permeability ratio whereas it is suppressed with thermal conductivity ratio. They also showed that Darcy number significantly influences the average Nusselt number. Kumar and Topin [21] investigated the influence of flow properties of the geometrical characteristics of metal foams in the porosity range of 80-95\% with a non-Darcy foam matrix model and 3-D direct numerical simulations at pore scale up to Reynolds numbers of 500. They computed the permeability in the Darcy regime, delineating this from the permeability associated with the inertia regime (Forchheimer effect), including good correlation with experimental results. Umavathi and Bég [22] deployed a Runge-Kutta quadrature and a shooting method to compute the thermosolutal dissipative convection flow in an open two-dimensional vertical channel containing a porous medium saturated with reactive Newtonian fluid and mixed (Robin) boundary conditions. They showed that Nusselt number at the right wall is depleted with higher thermal Grashof number, concentration (solutal) Grashof number and Brinkman number, whereas it is enhanced with 
chemical reaction parameter, porous medium (Darcian) parameter and Forchheimer inertial (quadratic drag) parameter; the opposite trends are observed at the left wall. They further showed that strong flow retardation is induced with Forchheimer drag. Sultana and Hyder [23] used a finite element method to investigate the natural convection in a porous medium wavy walled enclosure comprising two isothermal vertical wavy walls and two adiabatic top and base walls. They presented streamline and isotherm distributions for a Prandtl number of unities, Darcy number varying from 0.01 to infinity (purely fluid regime), Rayleigh numbers up to 10 million and various aspect and surface waviness ratios. Baytaş [24] analysed the steady state natural convection in a square non-Darcy porous enclosure using a two-temperature model of microscopic heat transfer (thermal non-equilibrium model) for the heat transfer between the fluid and the solid phases with heat generation present in the solid phase. Baytaş and Baytaş [25] studied the natural convection in a differentially heated enclosure with heat-generating porous non-Darcy layer on the inner walls for different internal and external porosity-scaled thermal conductivity ratio, Rayleigh numbers, Darcy numbers, solid-/fluid-scaled heat transfer coefficient and porous layer thickness. They observed that internal heat generation intensifies thermal convection for high values of the ratio of internal Rayleigh number to external Rayleigh number. Baytas et al. [26] examined the thermo-solutal convection between a saturated porous layer and an overlying fluid layer in a non-Darcian permeable enclosure (as a model of thermal insulation systems) for the cases where the interface contains a step and also when the interface between the fluid and porous layer is horizontal.

Rashad et al.,[51] studied double diffusive unsteady free convective liquid flow in porous medium inside a square cavity with distinct boundary conditions with an impaction of chemical reaction and thermal radiation, the thermal radiation enhancement results rise of Nusselt number and decrement of Sherwood number but an opposite phenomenon noticed in both the parameters when chemical reaction parameter raised. Rashad et al. [52] studied convective heat 
propagation of hybrid nanofluid packet in a triangular enclosure with uniform magnetic effect and heat flux at its bottom. While the inclined wall maintained cool and the rest of the side walls thermally-insulated. Based on heater size and its position impact of several parameters like Rayleigh number, Hartman number, volume fraction and heat generation parameters are investigated. A low-level natural convection observed for the rising values of volume fraction of the nanofluid. Mansour et al. [53] presented an analysis of entropy generation and MHD free convective $\mathrm{Cu}-\mathrm{Al}_{2} \mathrm{O}_{3}$ water nanofluid flow and heat expansion in a porous square enclosure heated and cooled differentially by source and sink. In their conclusion high rate of heat expansion observed when $\mathrm{Cu}$ included in the nanoparticles. Abdel-Nour et al. [54] studied MHD free convection of hybrid nanofluid (Al2O3-Cu/water) in a square cavity with porous medium. In their numerical results high heat transfer observed for the increment of Ra values and reverse phenomenon noticed for the rising values of Ha. For high Ra and Ha values flow cell also strengthened. Rashad et al. [55] Studied MHD free convection and heat propagation of $\mathrm{Cu}$-water nanofluid with in an inclined square enclosure under porous medium with an impact of heat source, sink and location. Investigation results reveals decrement of Nusselt number for the rising values of Hartman number and nanofluid volume fraction. The best heat source, sink and locations are also identified with the consideration of thermal and magnetic effects. Ahmed et al. [56] studied MHD free convection nanofluid by considering two heating modes within a fined triangular cavity with porous field. Based on the height, location and width of the fin impact of parameters like Hartmann number, nanoparticle volume fraction, heat generation/absorption parameters on fluid motion and heat transfer are studied. Fluid flow decreased with the increment of height of the fin in addition to this heat transfer also raised with the increment of height and width of the fin. Ahmed [57] discussed mixed convection flow in in a non-Darcy porous enclosure with bejan's heatlines approach. They are found that, for the low values of the Richardson number, the forced convection plays a dominant role in 
the flow region. Ahmed et al. [58] addressed significance of heat generation on free convection and heat transfer of nanofluids flow inside wavy enclosures filled with saturated porous medium. They are found that the heat transfer rate is an increasing function in both undulation number and the wavy contraction ratio. Mansour et al. [59] studied numerical investigation of laminar mixed convection cooling of heat source embedded on the bottom wall of an enclosure filled with nanofluids.

In recent years with the thrust for sustainable energy and fuel systems, engineers have explored a variety of different technologies for fuel cells. These include non-flammable gases e.g. helium and magnetohydrodynamics (MHD) which involves the interaction of applied magnetic fields with viscous electrically conducting fluids. Although significant progress has been made in hydrogen fuel cells; however, it has the disadvantage of being reactive and flammable and can lead to serious corrosion problems for enclosure boundaries. Helium is however very unreactive, can be deployed to achieve an inert atmosphere for delicate situations and is also non-toxic. Li et al. [27] explored the use of helium fluidized fuel cells in direct carbon conversion. They observed good current-voltage characteristics, impressive power densities and successful conversion of carbon in the flue stream. Magnetohydrodynamic effects in partially ionized helium have been confirmed by Devoto and Li [28]. Further study of the thermofluid characteristics of magnetic helium-based fuel cells is therefore of some interest. This is particularly important in terms of improving thermal management of existing fuel cell technologies [29, 30]. Important preliminary work in this regard has been communicated recently by Kanawkal and Othman [31] and much earlier by Guidotti [32]. The accurate performance of hydromagnetic fuel cells requires comprehensive laboratory testing and also numerical simulations of nonlinear viscous electrically conducting flows in enclosures and other geometries. Maqbool et al. [33] studied Lorentzian magnetic body force effects on unsteady rotating duct flows with a non-Newtonian working fluid and Fourier series. 
Magnetohydrodynamic flows in porous media have also garnered some attention with regard to hybrid fuel cell applications. Bég et al. [35] deployed an efficient finite difference technique (Keller's box method) to compute the hydromagnetic natural convection flow from a rotating metallic cone in anisotropic permeable media with a Darcy formulation. Jiang et al. [36] used a finite volume numerical method to simulate the natural thermomagnetic heat transfer of air in a porous cubic enclosure with an electric coil inclined around the vertical axis. They computed the mean Nusselt number on the hot boundary and showed that Lorentzian magnetic force and coil inclination significantly enhance heat transfer and damp the velocity. Begum et al. [37] used a finite difference method to study the free and forced magneto-convection in a non-Darcy porous medium enclosure saturated with copper-water nanofluid under oblique magnetic field. They showed that with increasing inclination and strength of the magnetic field, thermal convection is damped significantly. Iliuta and Larachi [38] computed the two-phase pressure drop and the total liquid holdup in hydromagnetic trickle bed reactors with a KozenyCarman approach. Alloui et al. [39] used a finite element code to compute the electromagnetic natural convection in a horizontal shallow porous cavity with uniform heat flux on the horizontal walls and adiabatic vertical walls. They studied the onset of convection and finite amplitude convection rolls for a variety of thermal Rayleigh numbers, Hartmann numbers, current intensity ratios and cavity aspect ratio.

In the present study we develope a MAC (marker and cell) algorithm [40] to simulate heat generating MHD flow in a square enclosure containing a high permeability non-Darcy porous medium saturated with electrically conducting Helium gas. A local thermal equilibrium (LTE) approach is used, and the Brinkman-Forchheimer extended Darcy model is employed for porous medium hydrodynamic drag effects $[37,41]$. The non-dimensional boundary value problem is solved in pressure-velocity form. Extensive visualizations of the impact of Grashof number $(G r)$, Hartmann magnetic number $(H a)$, Darcy number $(D a)$, and the internal heat 
generation parameter $(\Gamma)$ on streamline and isotherm distributions, with Prandtl number $(\operatorname{Pr})$ constrained as 0.71 (Helium) are presented. The variation in local Nusselt number at the left and right walls of the enclosure is also studied. Validation and grid-independence tests are included. Local Nusselt number is elevated at both left and right walls with greater Darcy number (higher medium permeability) and Grashof number. However, with greater internal heat generation, local Nusselt number magnitudes are enhanced at the left (cold) wall only but suppressed at the right (hot) wall. Increasing magnetic field reduces local Nusselt number at both left and right walls. With increasing magnetic field, the single vortex is strongly distorted and skewed towards the top left and lower right corners of the enclosure. Temperature contours at the left and right wall are however less intense with greater magnetic field effect. The simulations are of relevance to hybrid electromagnetic gaseous fuel cells, magnetic field control of filtration processes and porous media materials processing systems.

\section{ELECTROMAGNETIC CONVECTIVE NON-DARCY MODEL}

Fig. 1 represents the two-dimensional square enclosures with opposing thermal boundary conditions. The enclosure of the left (cold) and right (hot) walls are uniform temperatures, $T_{C}$ and $T_{H}$ respectively are imposed while the top and bottom walls are considered thermally insulated. The mathematical model is developed for the natural convective systems based on the following assumptions.

- Fluid is assumed to be incompressible and Newtonian.

- The no-slip boundary condition is assumed at the solid boundaries.

- The fluid flow is assumed to be laminar and two dimensional.

- The generalized form of the momentum balance equations based on the BrinkmanForchheimer extended Darcy model is considered. 
- The thermo-physical properties of the fluid except the density variation in the buoyancy term are considered to be constant. The Boussinesq approximation is invoked to relate the variation of density with temperature in the body force term.

- The temperature of the fluid phase is equal to the temperature of the solid phase in the case of the porous bed and the local thermal equilibrium (LTE) is applicable.

- Radiation, viscous dissipation and joule heating effects are negligible.

Bases on the above assumptions the equations (mass, primary momentum, secondary momentum and energy) can be shown to take the form [60]:

$\frac{\partial u}{\partial x}+\frac{\partial v}{\partial y}=0$

$\rho\left[\frac{\partial u}{\partial t}+u \frac{\partial u}{\partial x}+v \frac{\partial u}{\partial y}\right]=-p_{x}+\mu \nabla^{2} u-\frac{\mu}{K} u-F c \frac{\rho}{k^{1 / 2}} u \sqrt{u^{2}+v^{2}}$

$\rho\left[\frac{\partial v}{\partial t}+u \frac{\partial v}{\partial x}+v \frac{\partial v}{\partial y}\right]=-p_{y}+\mu \nabla^{2} v-\frac{\mu}{K} v-F c \frac{\rho}{k^{1 / 2}} v \sqrt{u^{2}+v^{2}}$

$$
-\sigma B_{0}^{2} v+\rho g \beta\left(T-T_{c}\right)
$$

$\rho C_{p}\left(\frac{\partial T}{\partial t}+u \frac{\partial T}{\partial x}+v \frac{\partial T}{\partial y}\right)=k \nabla^{2} T+q^{/ / /}$

Here $\nabla^{2}$ denotes the Laplacian operator. The prescribed boundary conditions at the four walls of the enclosure (spatial and temporal) are as follows:

$$
\begin{aligned}
& \text { At } t=0: u=v=T=0 \\
& u=v=0, T=T_{H} \quad x=L(\text { right wall }) \\
& u=v=0, T=T_{C} \text { at } x=0 \text { (left wall) } \\
& u=v=0, \frac{\partial T}{\partial y}=0 \text { at } y=0 \text { (base wall) } \\
& u=v=0, \frac{\partial T}{\partial y}=0, y=H(\text { upper wall })
\end{aligned}
$$


Proceeding with the analysis, a set of dimensionless parameters is invoked to normalize the nonlinear primitive boundary value problem defined by Eqns. (1)-(9). Defining:

$$
\begin{aligned}
& X=\frac{x}{H}, Y=\frac{y}{H}, U=\frac{\partial \psi}{\partial Y}=\frac{u H}{v}, V=-\frac{\partial \psi}{\partial X}=\frac{v H}{v}, \\
& \tau=\frac{t v}{H^{2}}, \theta=\frac{T-T_{0}}{T_{H}-T_{0}}, A=\frac{L}{H}, D a=\frac{K}{H^{2}}, G r=\frac{g \beta\left(T_{H}-T_{C}\right) H^{3}}{v^{2}}, T_{0}=\frac{T_{H}-T_{C}}{2} \\
& \operatorname{Pr}=\frac{v}{\alpha}, \Gamma=2 \frac{R a_{I}}{R a}, R a_{I}=\frac{g \beta q^{\prime \prime \prime} H^{5}}{k \alpha v}, H a=\frac{B_{0} H \sqrt{\sigma}}{\sqrt{\mu}}
\end{aligned}
$$

Introducing these transformations, the conservation equations with new dependent variables $U$,

$V, \theta$ emerge as follows, in vorticity formulation, with the Forchheimer (quadratic) drag coefficient prescribed as $F c=(1.75 / \sqrt{ } 150)$ [41], and mass conservation automatically satisfied:

\section{Primary Momentum}

$\frac{\partial U}{\partial \tau}+U \frac{\partial U}{\partial X}+V \frac{\partial U}{\partial Y}=-P_{X}+\nabla^{2} U-\frac{1}{D a} U-\frac{1.75}{\sqrt{150}} \frac{U \sqrt{U^{2}+V^{2}}}{\sqrt{D a}}$

\section{Secondary Momentum}

$$
\frac{\partial V}{\partial \tau}+U \frac{\partial V}{\partial X}+V \frac{\partial V}{\partial Y}=-P_{Y}+\nabla^{2} V-\frac{1}{D a} V-\mathrm{Ha}^{2} V-\frac{1.75}{\sqrt{150}} \frac{U \sqrt{U^{2}+V^{2}}}{\sqrt{D a}}+\frac{G r}{2} \theta
$$

\section{Energy}

$$
\frac{\partial \theta}{\partial \tau}+\frac{\partial(U \theta)}{\partial X}+\frac{\partial(V \theta)}{\partial Y}=\frac{1}{\boldsymbol{P r}} \nabla^{2} \theta+\frac{\Gamma}{\boldsymbol{P r}}
$$

The transformed conditions prescribed at the four boundaries now become (ignoring pressure terms):

$$
\begin{aligned}
& \text { At } \tau=0: U=V=\theta=0 \\
& U=V=0, \theta=-1 \text { at } X=0
\end{aligned}
$$


$U=V=0, \theta=1$ at $X=1$

$U=V=0, \frac{\partial \theta}{\partial Y}=0$ at $Y=0$ and $Y=1$

A local Nusselt number i.e. non-dimensional heat transfer rate at the boundary can be introduced. For the right (hot) vertical wall of the fuel cell enclosure this takes the form:

$\theta=\frac{T-T_{0}}{T_{H}-T_{0}}$

$N u=\frac{q H}{k\left(T_{H}-T_{C}\right)}=-\frac{1}{2}\left[\frac{\partial \theta}{\partial X}\right]_{X=1}$

The corresponding average Nusselt number, designated by $N u_{\mathrm{av}}$, may be computed as follows:

$$
N u_{a v}=-\frac{1}{2} \int_{0}^{1} \frac{\partial \theta}{\partial X} d Y
$$

\section{COMPUTATIONAL FINITE DIFFERENCE MAC SOLUTION}

The eqns. (11)-(17) are converted into conservative weak form and solved with a Harlow-

Welch MAC finite difference code. A stable, convergent solution is achieved utilizing a staggered grid system. The streamline patterns within the enclosure are employed with stream function and the projection method is adopted for the momentum equation. The stream function is computed with:

$U=\frac{\partial \psi}{\partial Y}, \quad V=-\frac{\partial \psi}{\partial X}$

This is solved by the well-known iterative Gauss Seidel method. Further details are given in [42]-[45]. Using the weak conservative form of the two-dimensional momentum and heat conservation equation a grid meshing procedure is deployed. Since a square enclosure is considered $(L=H$ i.e. aspect ratio $=1)$ a uniform structured mesh of density $80 \mathrm{X} 80$ is implemented which produces grid-independent solutions. 
First order explicit time integration discretization of present investigation as follows

Now Solve the intermediate velocity as follows

$$
\begin{aligned}
& U^{* * *}=U^{n}-\Delta t\left[-\left(U \frac{\partial U}{\partial X}+V \frac{\partial U}{\partial Y}\right)+\nabla^{2} U-\frac{1}{D a} U-\frac{1.75}{\sqrt{150}} \frac{U \sqrt{U^{2}+V^{2}}}{\sqrt{D a}}\right]^{n} \\
& V^{* * *}=V^{n}-\Delta t\left[\begin{array}{l}
-\left(U \frac{\partial V}{\partial X}+V \frac{\partial V}{\partial Y}\right)+\nabla^{2} V-\frac{1}{D a} V-\boldsymbol{H a}^{2} V-\frac{1.75}{\sqrt{150}} \frac{U \sqrt{U^{2}+V^{2}}}{\sqrt{D a}} \\
+\frac{G r}{2} \theta
\end{array}\right]
\end{aligned}
$$

After obtaining the intermediate velocities, we need to solve the following Pressure Poisson Equation (PPE)

The PEE equation is

$$
\nabla^{2} P^{n+1}=\frac{1}{\Delta t}\left(\frac{\partial U^{* *}}{\partial X}+\frac{\partial V^{* *}}{\partial Y}\right)
$$

The corrected velocities are obtained with the corrector step as given below

$$
\begin{gathered}
U^{n+1}=U^{* *}-\Delta t\left(\frac{\partial P^{n+1}}{\partial X}\right) \\
V^{n+1}=V^{* *}-\Delta t\left(\frac{\partial P^{n+1}}{\partial Y}\right)
\end{gathered}
$$

The temperature calculated as follows

$$
\theta^{n+1}=\theta^{n}-\Delta t\left[-\left(\frac{\partial(U \theta)}{\partial X}+\frac{\partial(V \theta)}{\partial Y}\right)+\frac{1}{\boldsymbol{P r}} \nabla^{2} \theta+\frac{\Gamma}{\boldsymbol{P r}}\right]^{n}
$$

Convergence criteria for computer codes is assumed to be 
$\left|\gamma^{n+1}-\gamma^{n}\right| \leq 10^{-8}$

where $\gamma$ could be any one of the non-primitive flow variables $(\mathrm{U}, \mathrm{V}, \mathrm{T}), \mathrm{n}$ denotes iteration umber. The following steps are employed to solve the discretized equations.

Step 1: To calculate the intermediate velocities we solve momentum equations eq. (21) and eq. (22) in discretized form first.

Step 2: Using the computed intermediate velocities, Successive Over-Relaxation (SOR) method is used to solve PPE eq. (23).

Step 3: The final velocity values can be obtained by the computed intermediate velocities and pressure in step 2 and step 3.

Step 4: Using the velocity values calculated in step 4, the thermal transport equations eq. (26)) in discretized for are solved to compute temperature in the computational region.

Verification of the accuracy of the MAC solutions is achieved by benchmarking with the nonmagnetic, non-porous (purely fluid) solutions available in Vahl Davis [46] (second order central finite difference technique), Manzari [47] (explicit finite element) and Wan et al. [48] (quasi-wavelet-based discrete singular convolution (DSC) technique with Shannon kernel and Galerkin finite element method). The comparisons are shown in Tables 1 and 2 and the associated data is $P r=0.71, H a=\Gamma=O$ (absence of magnetohydrodynamic effect and internal heat generation) and $D a \rightarrow \infty$ (infinite permeability i.e. purely fluid enclosure regime). Very good correlation is achieved between the MAC solutions with an 80X80 mesh and [46]-[48]. The grid differencing design $(80 \times 80)$ is visualized in Fig. 2.

\section{MAC NUMERICAL RESULTS FOR GENERAL MODEL}

A detailed parametric study of the influence of the influence of Grashof number $(\mathrm{Gr})$, Hartmann magnetic number $(H a)$, Darcy number $(D a)$, and internal heat generation parameter 
$(\Gamma)$ on streamline and isotherm distributions has been conducted. In all simulations Prandtl number $(\mathrm{Pr})$ is prescribed as 0.71 (electrically conducting Helium at 100 Celsius $[27,28])$. The contour plots are illustrated in Figs. 3 a, b -20 a, b. Additionally graphs for local Nusselt number distributions at the left and right walls of the enclosure are given in Figs. 21 a, b to 24

a, b. As noted earlier the Forchheimer coefficient is prescribed as $F c=\frac{1.75}{\sqrt{150}}$ which corresponds to high permeability, sparsely packed porous media [41].

Figs. 3a, b- 5a, b presents the contour plots for a variation in Grashof number $(G r)$. In all plots $G r>>0$ implying significant thermal convection currents are present in the enclosure fuel cell. For all $G r$ a solitary circulation zone is computed. Fig. 3a, b shows that there is a single symmetric vortex (circulation zone) and the isotherms are parallel to the vertical boundaries i.e. undistorted. With increasing Grashof number (Fig. 4a,b) the single cell becomes warped towards the upper left corner and lower right corners of the enclosure; the isotherms are also markedly skewed towards the upper region of the left wall (cold) i.e. they are non-parallel to the vertical walls. At highest Grashof number (Fig. 5a, b) the skewness in the single cell is exacerbated and streamlines are compressed further in the top left and lower right corners, expanding in the other two corners of the cavity. A much more prominent distortion in isotherms is observed with a constriction induced in the top left and lower right corners and a relaxation in the upper right and lower left corners. Increasing thermal buoyancy relative to viscous forces in the enclosure (Grashof number expresses the ratio of these two body forces) substantially modifies the vortex structure and heat distribution in the cavity. Asymmetrical behaviour is generated in both velocity and temperature fields in the electroconductive Helium gas with increasing thermal buoyancy, even in the absence of heat source effect $(\Gamma=0)$ and for very low permeability $(D a=0.0001)$. 
Figs. 6a, $\mathbf{b}-\mathbf{1 0 a}, \mathbf{b}$ displays the influence of internal heat source parameter $(I)$ on streamline and isotherm distributions. With weak heat generation $(\Gamma=1)$ i.e. in Fig. $6 a$, b, a slight warping of the single vortex cell towards the upper left enclosure corner is observed and some distortion in isotherms again towards the upper left corner. Isotherms are more closely clustered towards the cold left wall and become increasingly dispersed as we progress towards the right wall (hot). With increasing heat source effect $(\Gamma=2)$ i.e. in Fig. $7 \mathrm{a}, \mathrm{b}$, there is a progressive skewing in the single vortex towards the upper left corner and streamlines are denser towards the left cold wall. Isotherms also intensify towards the left wall and expand notably in the right half space of the enclosure, distorting increasingly towards the upper left corner. This pattern is further amplified with $\Gamma=3$ i.e. in Fig. 8 a, b, leading to a slight elongation in the vortex in the vertical direction and vanishing isotherms in the vicinity of the right hot wall. With $\Gamma=4$ i.e. in Fig. 9 a, b, the single vortex is again expanded vertically, warped further diagonally and the isotherm closest to the right hot wall bifurcates. The isotherm bifurcation is further developed for $\Gamma=5$ i.e. in Fig. $10 \mathrm{a}, \mathrm{b}$ and the single cell is increasingly compressed towards the left cold wall. Heat generation therefore exerts a considerable influence on thermal and momentum characteristics of the electrically conducting Helium in the fuel cell enclosure.

Figs. 11a, b - 14 a, b illustrates the streamline and isotherm distributions for various Darcy number (Da). Although Forchheimer drag is present, it is not explicitly varied and all porous media drag effects are simulated via Darcy number (which appears both in the Darcian linear drag and quadratic drag denominators i.e. $-\frac{1}{D a} U,-\frac{1.75}{\sqrt{150}} \frac{U \sqrt{U^{2}+V^{2}}}{\sqrt{D a}}$ terms in the primary momentum Eqn. (11) and $-\frac{1}{D a} V,-\frac{1.75}{\sqrt{150}} \frac{U \sqrt{U^{2}+V^{2}}}{\sqrt{D a}}$ terms in the secondary momentum Eqn. (12)). Clearly as $D a$ is enhanced all drag components are reduced since the matrix offers progressively decreasing impedance to the Helium gas flow. With increasing Darcy number (i.e. a tenfold rise in permeability) from $D a=0.0001$ (Fig. 11a, b) to 0.001 (Fig. 12a, b) a 
strong distortion is computed in both isovels (streamlines) and isotherms. The single cell is stretched along the diagonal from the top left corner to the lower right corner of the enclosure i.e. is skewed and asymmetric. Isotherms are also and biased towards the left cold wall with closer clustering in this vicinity and an expansion away from the right hot wall. However, with subsequent increase in $\mathrm{Da}$ (Fig. $13 \mathrm{a}, \mathrm{b}$ ) to 0.01 the single vortex is elongated in the $\mathrm{x}$-direction i.e. stretched laterally, although it is larger towards the cold left wall. A stronger compression of isotherms is observed towards the left cold wall and a sigmodial distribution emerges from the top left to the lower right enclosure corners. Isotherms become significantly separated in the central core of the enclosure although some clustering appears towards the right hot wall. At highest Darcy number (Fig. 14a, b) with $D a=0.1$, there is however a substantial modification in the skewness of the vortex cell which is distorted now towards the lower left corner and upper right corner; it is strongly asymmetric with a larger zone towards the lower left wall and a constricted tilted zone towards the upper right wall. Isotherms are increasingly sigmoidal in topology and expand in the central core with intensification at the left cold and right walls. The boundary layer structure is significantly modified at the right and left walls with greater permeability effect. This is also characteristic of Forchheimer effects as noted by Dybbs and Edwards [49]. The Brinkman extended Darcy-Forchheimer model also captures vorticity diffusion effects at the boundaries. The pore scale convective inertial effects contributing to the form drag lead to a substantial alteration of the velocity field and exacerbate the macroscopic region in which the pore scale velocity gradients are large. Forchheimer drag simulates the strong inertial flow regime in porous media hydrodynamics. This pertains to the regime where the pore Reynolds number based on a consideration of the particle or pore diameter, is greater than or equal to unity. At this point the model departs from the purely Darcian or viscous-dominated classical porous media transport physics. It is also pertinent to 
note that for the value of $F c$ specified, vortex formation associated with higher Reynolds numbers (greater than 250) has not been considered.

Figs. 15a, $\mathbf{b}-\mathbf{2 0 a}, \mathbf{b}$ visualizes the impact of magnetic body force parameter on streamline and isotherm distributions. The Lorentzian magnetic body force, $-\mathrm{Ha}^{2} \mathrm{~V}$, arises only in the secondary momentum Eqn. (12) and acts in the reverse $y$ direction, since the magnetic field acts along the $x$-axis. Hartmann Number, $H a=\frac{B_{0} H \sqrt{\sigma}}{\sqrt{\mu}}$ signifies the relative contribution of Lorentzian magnetic drag force to viscous hydrodynamic force. In all the plots $H a>1$ i.e., magnetic force exceeds viscous resistance. At $H a=5$ (Fig. 15a, b) an asymmetrical laterally elongated single cell is computed with a larger zone towards the left wall and a narrower zone towards the right wall. Isotherms are also sigmoidal in nature from the top left corner to the lower right corner and some clustering is present at the left and right walls. With progressive increase in $\mathrm{Ha}$ to 10 (Fig. 16a, b), 25 (Fig. 17a, b) there is a warping in the vortex towards the upper left corner and lower right corner and the asymmetry is reduced somewhat. With yet greater magnetic field intensity for $H a=30$ (Fig. 18a, b), 35 (Fig. 19a, b) and 50 (Fig. 20a, b) the vortex is increasingly stretched in the diagonal direction from the upper left to the lower right corner and symmetrical in nature. The isotherms at the left and right walls become increasingly parallel to these boundaries and expand in the core region. With greater magnetic field, a larger amount of work has to be utilized to drag the electroconductive Helium gas against the action of the vertical Lorentzian drag force. This supplementary work used is dissipated as thermal energy and this elevates temperatures in the cavity.

Figs. 21a, b- 24a, b displays the distributions of local Nusselt number $(\mathrm{Nu})$ with vertical coordinate at both the left (cold) and right (hot) walls with all key parameters again for the case of Prandtl number $\operatorname{Pr}=0.71$ (ionized Helium). As with all other graphs the thermal diffusivity therefore exceeds the momentum diffusivity in the regime $(\operatorname{Pr}<1)$. Figs. 21a, b shows that 
with higher Grashof number i.e. increasing thermal buoyancy (natural convection) effect, although $N u$ is initially reduced for small values of $Y$ (i.e. closer to the base wall), some distance upwards along the cold left wall, a significant increase in local Nusselt number is generated. Conversely at the right hot wall, although there is a strong elevation in local Nusselt number for most of the distance along the wall, at high $Y$ values this trend is reversed and Nusselt number is found to be slightly reduced. At low Grashof number $\left(G r=2 \times 10^{4}\right)$ the local Nusselt number is constant along both left and right walls i.e. unaffected by location along the wall. Figs. 22 a, bindicate, that greater Hartmann number, $H a$, i.e. increasing magnetic field reduces local Nusselt number at both left and right walls. However, while $N u$ is a maximum at the top of the left wall, it is a minimum at the top of the right wall. Maximum $N u$ is observed at the base of the right hot wall and minimum $N u$ computed at the base of the left cold wall. All maxima correspond to the electrically non-conducting Helium gas case i.e. $H a=0$. Heat transfer rates to the left and right walls are therefore differently influenced with increasing magnetic field strength. Although heating is induced within the enclosure with stronger magnetic field (see Figs. 15a, b-20a, b), this manifests in a suppression of thermal energy migrating to both vertical boundaries. Figs. 23a, b demonstrates that, with greater internal heat generation, local Nusselt number magnitudes are enhanced at the left (cold) wall only but suppressed at the right (hot) wall. These patterns are sustained for all locations along either wall. Finally Figs. 24 a,b show that with greater Darcy number (higher medium permeability), although there is an initial reduction in $N u$ at the left cold wall, this is quickly superseded by a very strong elevation in local Nusselt number, a short distance from the base of the enclosure. Local Nusselt number at the right hot wall, although initially decreased near the top of the right wall, is subsequently considerably enhanced with increasing Darcy number at all other locations along the wall all the way to the enclosure base. Permeability of the enclosure fuel cell therefore substantially 
modifies the heat transfer rate to the vertical boundaries, and this may be exploited in fuel cell designs $[27,29,30]$.

\section{CONCLUSIONS}

Motivated by exploring new hybrid designs for magnetohydrodynamic (MHD) ionized gas porous media fuel cells, an analytical and numerical study of natural magneto-convection in an isotropic non-Darcian porous medium saturated with electrically conducting Helium in a twodimensional enclosure with heat source is presented. A Brinkman-extended DarcyForchheimer model is employed and the medium is assumed to be homogenous and nondeformable. The non-dimensional mass and primary and secondary momentum conservation equations and boundary conditions imposed at the four sides of the enclosure are converted to vorticity form and solved with a Harlow-Welch marker and cell (MAC) finite difference technique. Verification of the accuracy of the MAC code is achieved with comparison with previous studies for air in the absence of magnetic field and porous media effects. Meshindependence tests are also conducted. The simulations have shown that:

(i) With increasing Grashof number (thermal buoyancy) the skewness in the single vortex cell is amplified and streamlines are compressed further in the top left and lower right corners, expanding in the other two corners of the cavity. A strong distortion in isotherms is also observed with a constriction induced in the top left and lower right corners and a relaxation in the upper right and lower left corners. Furthermore, local Nusselt number is elevated at both left and right walls with higher Grashof number.

(ii) With greater internal heat generation, the single vortex is expanded vertically, and the isotherm closest to the right hot wall bifurcates, with clustering of isotherms 
towards the left cold wall. Local Nusselt number magnitudes are enhanced at the left (cold) wall whereas they are reduced at the right (hot) wall and this behaviour at both walls is sustained for all locations.

(iii) At lower Darcy number (low medium permeability) the single cell is stretched along the diagonal from the top left corner to the lower right corner of the enclosure i.e. is skewed and asymmetric and isotherms are also and biased towards the left cold wall with closer clustering in this vicinity. At high Darcy number however the skewness of the vortex cell is adjusted towards the lower left corner and upper right corner; it is strongly asymmetric with a larger zone towards the lower left wall and a constricted zone towards the upper right wall. Isotherms are increasingly sigmoidal in topology at higher Darcy number and expand in the central core with intensification at the left cold and right walls and Forchheimer effects markedly modify the boundary layer structure at both vertical boundaries. Increasing Darcy number (higher medium permeability), initially decreases local Nusselt number at the left cold wall (closer to the base of the enclosure), but shortly thereafter produces a considerable elevation in local Nusselt number. Local Nusselt number at the right hot wall, although initially decreased near the top of the right wall, is subsequently considerably enhanced with increasing Darcy number at all other locations along this wall.

(iv) At lower Hartmann numbers (weaker magnetic field), an asymmetrical laterally elongated single cell is computed with a larger zone towards the left wall and a narrower zone towards the right wall. Isotherms are also sigmoidal in nature from the top left corner to the lower right corner and some clustering is present at the left and right walls. However, with higher Hartmann number, a significant distortion is induced in the vortex cell towards the upper left corner and lower right corner and 
the asymmetry is reduced; isotherms are also intensified at the left wall with stronger magnetic field. Increasing magnetic field additionally depletes local Nusselt number at both left and right walls.

(v) The present MAC code has revealed some interesting thermal fluid characteristics for hybrid fuel cells. However, attention has been confined to rigid boundaries.

Future studies may examine wavy sinusoidal boundaries and efforts in this regard will be communicated imminently. 


\section{REFERENCES}

[1] S. Singh. Experimental and numerical investigations of a single and double pass porous serpentine wavy wire mesh packed bed solar air heater. Renewable Energy.2020; 145: 13611387.

[2] N. Dukhan. Analysis of Brinkman-extended Darcy flow in porous media and experimental verification using metal foam. ASME J. Fluids Eng 2012; 134 (7): 071201.

[3] O. Anwar Bég, Sandile S. Motsa, A. Kadir, et al. Islam, Spectral quasilinear numerical simulation of micropolar convective wall plumes in high permeability porous media. $J$. Engineering Thermophysics 2016; 25 (4):1-24.

[4] W. Choi and R. Ooka. Effect of natural convection on thermal response test conducted in saturated porous formation: Comparison of gravel-backfilled and cement-grouted borehole heat exchangers. Renewable Energy 2016; 96: 891-903.

[5] O. Anwar Bég, S.S. Motsa, Eemaan T. A. Bég, et al. Numerical study of nonlinear heat transfer from a wavy surface to a high permeability medium with pseudo-spectral and smoothed particle methods. Int. J. Applied Computational Mathematics 2017; 3: 3593-3613.

[6] J. Belabid. Impact of wall waviness on the convection patterns inside a horizontal porous annulus. ASME J. Fluids Eng 2020; 142(7): 071304.

[7] Vafai, K., Porous Media: Applications in Biological Systems and Biotechnology, CRC Press, Boca Raton, Florida 2010.

[8] D. Tripathi and O. Anwar Bég. A numerical study of oscillating peristaltic flow of generalized Maxwell viscoelastic fluids through a porous medium. Transport in Porous Media 2012; 95: 337-348. 
[9] O. Anwar Bég, V.R. Prasad, B. Vasu. Numerical study of mixed bioconvection in porous media saturated with nanofluid and containing oxytactic micro-organisms. J. Mechanics Medicine Biology 2013; 13: 1350067.1-1350067.25.

[10] Caltagirone, J. P. Thermoconvective instabilities in a porous medium bounded by two concentric horizontal cylinders. J. Fluid Mech 1976; 76: 337 -362.

[11] M.L. Martins-Costa et al. modelling and simulation of natural convection flow in a saturated porous cavity, Meccanica. 1994; 29: 1-13.

[12] H. Saleh and I. Hashim, Conjugate natural convection in a porous enclosure with nonuniform heat generation, Transport in Porous Media 2012; 94: 759-774.

[13] P. G. Siddheshwar and C. Siddabasappa, Unsteady natural convection in a liquid-saturated porous enclosure with local thermal non-equilibrium effect, Meccanica 2020; 55: 1763-1780.

[14] Basak, Tanmay, S. Roy, and S. Krishna Babu, Natural convection and flow simulation in differentially heated isosceles triangular enclosures filled with porous medium, Chemical Engineering Science 2008; 63: 3328-3340.

[15] Chen, Y., Li, B., Zhang, J. Spectral collocation method for natural convection in a square porous cavity with local thermal equilibrium and non-equilibrium models. Int. J. Heat Mass Transfer 2016; 96: 84-96.

[16] Song, M., Viskanta, R. Natural convection flow and heat transfer within a rectangular enclosure containing a vertical porous layer. Int. J. Heat Mass Transfer 1994; 37(16): 24252438.

[17] O. Anwar Bég, M.J. Uddin, T. Bég. Numerical simulation of self-similar thermal convection from a spinning cone in anisotropic porous medium,J. Hydrodynamics Series $B$ 2016; 28 (2): 184-194. 
[18] A. E. Scheidegger, Physics of Flow through Porous Media, Macmillan Book Co., New York (1960).

[19] T. A. Bég, M.M. Rashidi, O. Anwar Bég and N. Rahimzadeh, Differential transform seminumerical simulation of biofluid-particle suspension flow and heat transfer in non-Darcian porous media, Computer Methods Biomechanics Biomedical Engineering, 2013; 16 (8): 896907.

[20] P. Chandra and V.V. Satyamurty, Non-Darcian and anisotropic effects on free convection in a porous enclosure, Transport in Porous Media 2011; 90: 301-320.

[21] P. Kumar and F. Topin, Investigation of fluid flow properties in open cell foams: Darcy and weak inertia regimes, Chemical Engineering Science 2014; 116: 793-805.

[22] J.C. Umavathi and O. Anwar Bég, Numerical study of double-diffusive dissipative reactive convective flow in an open vertical duct containing a non-Darcy porous medium with Robin boundary conditions, Journal of Engineering Mathematics 2019; 119: 135-147.

[23] Z. Sultana and M.N. Hyder, Non-Darcy free convection inside a wavy enclosure, International Communications in Heat and Mass Transfer 2007; 34: 136-146.

[24] A.C. Baytaş, Thermal non-equilibrium natural convection in a square enclosure filled with a heat-generating solid phase, non-Darcy porous medium, International Journal of Energy Research 2013; 27 (10): 975-988.

[25] A. Filiz Baytaş and A. CihatBaytaş, Thermal non-equilibrium natural convection in a square enclosure with heat-generating porous layer on inner walls, Transport in Porous Media 2017; 120: 167-182. 
[26] Baytaş, A.C., Baytaş, A.F., Ingham, D.B., Pop, I., Double diffusive natural convection in an enclosure filled with a step type porous layer: non-Darcy flow. Int. J. Therm. Sci. 2009; 48(4): 665-673.

[27] S. Li et al., Direct carbon conversion in a helium fluidized bed fuel cell, Solid State Ionics 2008; 179: 1549-1552.

[28] R. S. Devoto and C. P. Li, Transport coefficients of partially ionized helium, J. Plasma Physics 1968; 2 (1): 17-32.

[29] Faghri A, Guo Z., Challenges and opportunities of thermal management issues related to fuel cell technology and modeling. International Journal of Heat and Mass Transfer 2005; 48: 3891-3920.

[30] Bao C, Ouyang M, Yi B., Analysis of the air and thermal management in insulated heat exchange membrane fuel cell systems. International Journal of Hydrogen Energy 2006; 31: 1040-1057.

[31] K. Kanawka and M. H. D. Othman, NI/NI-YSZ Current collector/anode dual layer hollow fibers for micro-tubular solid oxide fuel cells, Fuel Cells 2011; 11: 690-696.

[32] R. Guidotti, Evaluation of MHD materials for use in high-temperature fuel cells, Topical Report I - SAN-1485-1, The Montana Energy and MHD Research and Development Institute, Inc. 'Bune, Montana, USA,1978;

[33] V. C. A. Ferraro and C. Plumpton, An Introduction to Magneto-Fluid Mechanics, Oxford University Press, USA 1961.

[34] K. Maqbool, O. Anwar Bég, Ayesha Sohail, ShafaqIdreesa, Analytical solutions for magnetohydrodynamic oscillatory rotating plate and channel flows in porous media using a fractional Burgers viscoelastic model, European Physical Journal Plus 2016; 131: 140-157. 
[35] O. Anwar Bég, B. Vasu and R.S.R. Gorla, Computational modelling of magnetohydrodynamic convection from a rotating cone in orthotropic Darcian porous media, J. Braz. Society Mech Sci Eng 2017; 39: 2035-2054.

[36] C. Jiang et al., Magnetic and gravitational convection of air in a porous cubic enclosure with a coil inclined around the Y axis, Transport in Porous Media 2014; 102: 167-183.

[37] A.S. Begum et al., Numerical simulation of MHD mixed convection in a nanofluid filled non-Darcy porous enclosure, International Journal of Mechanical Sciences, 2017; 130: 154166.

[38] I. Iliuta and F. Larachi, Magnetohydrodynamics of trickle bed reactors: Mechanistic model, experimental validation and simulations, Chemical Engineering Science 2003; 58 (2): 297-307.

[39] Z. Allouiet al., Effect of a non-constant magnetic field on natural convection in a horizontal porous layer heated from the bottom, J. Engineering Math 2013; 81: 141-155.

[40] F.H. Harlow, The marker-and-cell method, Numerical Methods in Fluid Dynamics, Von Karman Inst. for Fluid Dynamics, Belgium, 1972; 38.

[41] M. Siavashi, V. Bordbar, P. Rahnama, Heat transfer and entropy generation study of nonDarcy double-diffusive natural convection in inclined porous enclosures with different source configurations, Applied Thermal Engineering 2017; 110: 1462-1475.

[42] K. Venkatadri, S. Abdul Gaffar, V. R. Prasad, Simulation of natural convection heat transfer in a 2-D trapezoidal enclosure, International Journal of Automotive and Mechanical Engineering 2019; 16 (4): 7375-7390. 
[43] K. Venkatadri, O. Anwar Bég, V. R. Prasad, Numerical simulation of thermal radiation influence on natural convection in a trapezoidal enclosure: heat flow visualization through energy flux vectors, Int. J. Mechanical Sciences 2020; 171.

[44] O. Anwar Bég, K. Venkatadri, V.R. Prasad, Numerical simulation of hydromagnetic Marangoni convection flow in a Darcian porous semiconductor melt enclosure with buoyancy and heat generation effects, Materials Science and Engineering B, 202; 261: 114722.

[45] Tannehill, J.C., Anderson, D.A., and Pletcher, R. H., Computational Fluid Mechanics and Heat Transfer, Taylor \& Francis Ltd., USA 1997.

[46] D. de Vahl Davis, Natural convection of air in a square cavity: a benchmark solution, Int. J. Num. Meth. Fluids 1983; 3; 249-264.

[47] M. T. Manzari, An explicit finite element algorithm for convective heat transfer problems, Int. J. Numer. Meth. Heat Fluid Flow 1999; 9; 860- 877.

[48] D. C. Wan, B. S. V. Patnaik, and G. W. Wei,A new benchmark quality solution for the buoyancy-driven cavity by discrete singular convolution, Numerical Heat Transfer:Part B, 2001; 40: 199-228.

[49] A. Dybbs and R. V. Edwards, A new look at porous media fluid mechanics - Darcy to turbulent, Fundamentals of Transport Phenomena in Porous Media_pp 199-256, Ed. J. Bear, NATO Series, Netherlands 1984.

[50] M.J. Uddin, W.A. Khan, and O. Anwar Bég, Bioconvection nanofluid slip flow past a wavy surface with applications in nano-biofuel cells Chin. J. Physics 2017; 55: 2048-2063.

[51] Rashad, A. M., Elsayed Ahmed, S., \& Ahmed Mansour, M., Effects of chemical reaction and thermal radiation on unsteady double diffusive convection. International Journal of Numerical Methods for Heat \& Fluid Flow 2014; 24(5): 1124-1140. 
[52] Rashad, A. M., Chamkha, A. J., Ismael, M. Magnetohydrodynamics Natural Convection in a Triangular Cavity Filled with a Cu-Al2O3/Water Hybrid Nanofluid With Localized Heating from Below and Internal Heat Generation. Journal of Heat Transfer, 2018; 140(7): 072502.

[53] Mansour, M. A., Siddiqa, S., Gorla, R. S. Effects of heat source and sink on entropy generation and MHD natural convection of $\mathrm{Al} 2 \mathrm{O} 3-\mathrm{Cu} /$ water hybrid nanofluid filled with square porous cavity. Thermal Science and Engineering Progress 2018; 6: 57-71.

[54] Abdel-Nour, Z., Aissa, A., Mebarek-Oudina,. Magnetohydrodynamic natural convection of hybrid nanofluid in a porous enclosure: numerical analysis of the entropy generation. Journal of Thermal Analysis and Calorimetry. 2020.

[55] Rashad, A. M., Armaghani, T., Chamkha, A. J. Entropy generation and MHD natural convection of a nanofluid in an inclined square porous cavity: Effects of a heat sink and source size and location. Chinese Journal of Physics 2018; 56(1): 193-211.

[56] Ahmed, S. E., Mansour, M. A., Rashad, MHD natural convection from two heating modes in fined triangular enclosures filled with porous media using nanofluids. Journal of Thermal Analysis and Calorimetry. 2019.

[57] S.E. Ahmed, Mixed convection in thermally anisotropic non-Darcy porous medium in double lid-driven cavity using Bejan's heatlines, Alexandria Engineering Journal, 2016; 55: 299-309.

[58] S.E. Ahmed, Z.Z. Rashed, MHD natural convection in a heat generating porous mediumfilled wavy enclosures using Buongiorno's nanofluid model, Case Studies in Thermal Engineering 2019; 14: 100430. 
[59] M.A. Mansour, S.E. Ahmed, Mixed convection flows in a square lid-driven cavity with heat source at the bottom utilising nanofluid, The Canadian Journal of Chemical Engineering, 2012; 90: 100-110.

[60] Ahmed, S.E. Non-Darcian natural convection of a nanofluid due to triangular fins within trapezoidal enclosures partially filled with a thermal non-equilibrium porous layer. $J$ Therm Anal Calorim 2020. 


\section{Graphical abstract}



\section{FIGURE CAPTIONS.}

Fig 1: Physical model for hybrid electromagnetic Helium porous medium fuel cell enclosure

Figure 2: Uniform finite difference mesh for square enclosure (hybrid fuel cell geometry)

Fig. 3 a) Streamlines and b) Isotherms for $\mathrm{Gr}=2 \times 10^{4}, \mathrm{Pr}=0.71 ; \mathrm{Ha}=5 ; \mathrm{Da}=0.0001$ and $\Gamma=0$

Fig. 4 a) Streamlines and b) Isotherms for $G r=2 \times 10^{5}, \mathrm{Pr}=0.71 ; \mathrm{Ha}=5 ; \mathrm{Da}=0.0001$, and $\Gamma=0$

Fig. 5 a) Streamlines and b) Isotherms for $G r=2 X 10^{6}, P r=0.71, H a=5, D a=0.0001$ and $\Gamma=0$

Fig 6 a) Streamlines and b) Isotherms for $G r=2 X 10^{5}, \mathrm{Pr}=0.71, \mathrm{Ha}=5, \mathrm{Da}=0.0001$ and $\Gamma=1$

Fig. 7a) Streamlines and b) Isotherms for $G r=2 X 10^{5}, \mathrm{Pr}=0.71, \mathrm{Ha}=5, \mathrm{Da}=0.0001$ and $\Gamma=2$

Fig. 8 a) Streamlines and b) Isotherms for $G r=2 X 10^{5}, P r=0.71, H a=5, D a=0.0001$ and $\Gamma=3$

Fig. 9 a) Streamlines and b) Isotherms for $G r==2 X 10^{5} ; \operatorname{Pr}=0.71, H a=5, D a=0.0001$, and $\Gamma=4$

Fig. 10a) Streamlines and b) Isotherms for $G r=2 X 10^{5} ; \mathrm{Pr}=0.71$; $\mathrm{Ha}=5 ; \mathrm{Da}=0.0001$ and $\Gamma=5$

Fig 11a) Streamlines and b) Isotherms for $G r=2 X 10^{5}, \operatorname{Pr}=0.71, \mathrm{Ha}=5, \Gamma=1$ and $\mathrm{Da}=$ 0.0001 . 
Fig 12 a) Streamlines and b) Isotherms for $G r=2 X 10^{5}, \operatorname{Pr}=0.71, \mathrm{Ha}=5, \Gamma=1$ and $\mathrm{Da}=$ 0.001

Fig 13a) Streamlines and b) Isotherms for $G r=2 X 10^{5}, \mathrm{Pr}=0.71, \mathrm{Ha}=5, \Gamma=1$ and $\mathrm{Da}=$ 0.01

Fig 14a) Streamlines and b) Isotherms for $G r=2 \times 10^{5}, \operatorname{Pr}=0.71, \mathrm{Ha}=5, \Gamma=1$ and $\mathrm{Da}=$ 0.1

Fig 15a) Streamlines and b) Isotherms for $\mathrm{Gr}=2 X 10^{5}, \mathrm{Pr}=0.71, \Gamma=1, \mathrm{Da}=0.01$ and $\mathrm{Ha}$ $=5$

Fig 16 a) Streamlines and b) Isotherms for $\mathrm{Gr}=2 X 10^{5}, \mathrm{Pr}=0.71, \Gamma=1, \mathrm{Da}=0.01$ and $\mathrm{Ha}$ $=10$

Fig 17a) Streamlines and b) Isotherms for $\mathrm{Gr}=2 X 10^{5}, \mathrm{Pr}=0.71, \Gamma=1, \mathrm{Da}=0.01$ and $\mathrm{Ha}$ $=25$

Fig 18a) Streamlines and b) Isotherms for $\mathrm{Gr}=2 X 10^{5}, \mathrm{Pr}=0.71, \Gamma=1, \mathrm{Da}=0.01$ and $\mathrm{Ha}$ $=30$

Fig 19a) Streamlines and b) Isotherms for $\mathrm{Gr}=2 X 10^{5}, \mathrm{Pr}=0.71, \Gamma=1, \mathrm{Da}=0.01$ and $\mathrm{Ha}$ $=35$

Fig 20a) Streamlines and b) Isotherms for $\mathrm{Gr}=2 X 10^{5}, \mathrm{Pr}=0.71, \Gamma=1, \mathrm{Da}=0.01$ and $\mathrm{Ha}$ $=50$

Fig. 21 Local Nusselt number for a) left cold wall, b) right hot wall with $\operatorname{Pr}=0.71, \mathrm{Ha}=5$, Da $=0.0001, \Gamma=1$ for different Grashof (thermal buoyancy) numbers $(G r)$

Fig. 22 Local Nusselt number for a) left cold wall, b) right hot wall with $G r=2 X 10^{5}, \operatorname{Pr}=$ 0.71, Da $=0.01, \Gamma=1$ for different Hartmann magnetic numbers $(\mathrm{Ha})$ 
Fig. 23 Local Nusselt number for a) left cold wall, b) right hot wall with $\operatorname{Pr}=0.71, \mathrm{Ha}=5$, $D a=0.0001, G r=2 X 10^{5}$ for different internal heat source parameters $(\Gamma)$

Fig. 24 Local Nusselt number for a) left cold wall, b) right hot wall with $\operatorname{Pr}=0.71, \mathrm{Ha}=5, \Gamma$ $=1, G r=2 \times 10^{5}$ for different Darcy numbers $(\mathrm{Da})$ 


\section{TABLES}

Table. 1. Grid independence and validation for Average Nusselt number $(N u)$ with $\operatorname{Pr}=0.71$

\begin{tabular}{|c|c|c|c|c|c|c|c|c|}
\hline $\mathrm{Ra}$ & $N u$ & $\begin{array}{c}\text { Ref. [46] } \\
\text { (central } \\
\text { finite } \\
\text { differences) }\end{array}$ & $\begin{array}{l}\text { Ref. [47] } \\
\text { (explicit } \\
\text { finite } \\
\text { elements) }\end{array}$ & $\begin{array}{c}\text { Ref. [48] } \\
\text { FEM }\end{array}$ & $\begin{array}{l}\text { Present } \\
\text { study } \\
40 X 40\end{array}$ & $\begin{array}{l}\text { Present } \\
\text { study } \\
80 \times 80\end{array}$ & $\begin{array}{c}\text { Present } \\
\text { study } \\
120 X 120\end{array}$ & $\begin{array}{l}\text { Present } \\
\text { study } \\
160 \times 160\end{array}$ \\
\hline $10^{4}$ & Average & 2.243 & 2.084 & 2.254 & 2.2526 & 2.2455 & 2.2444 & 2.2442 \\
\hline
\end{tabular}

Table. 2. Comparison of Average Nusselt number $(\mathrm{Nu})$ with $\operatorname{Pr}=0.71$

\begin{tabular}{cccccc}
\hline$R a$ & $N u$ & $\begin{array}{c}\text { Ref. [46] } \\
\text { (central finite } \\
\text { differences) }\end{array}$ & $\begin{array}{c}\text { Ref. [47] } \\
\text { (explicit finite } \\
\text { elements) }\end{array}$ & $\begin{array}{c}\text { Ref. [48] } \\
\text { FEM } \\
\text { (discrete singular } \\
\text { convolution and } \\
\text { Galerkin method) }\end{array}$ & (MAC) \\
\hline $10^{3}$ & Average & 1.12 & 1.074 & 1.117 & 1.1185 \\
$10^{4}$ & Average & 2.243 & 2.084 & 2.254 & 2.2526 \\
$10^{5}$ & Average & 4.52 & 4.3 & 4.598 & 4.5907 \\
$10^{6}$ & Average & 8.8 & 8.743 & 8.976 & 8.9905 \\
\hline
\end{tabular}




\section{FIGURES}

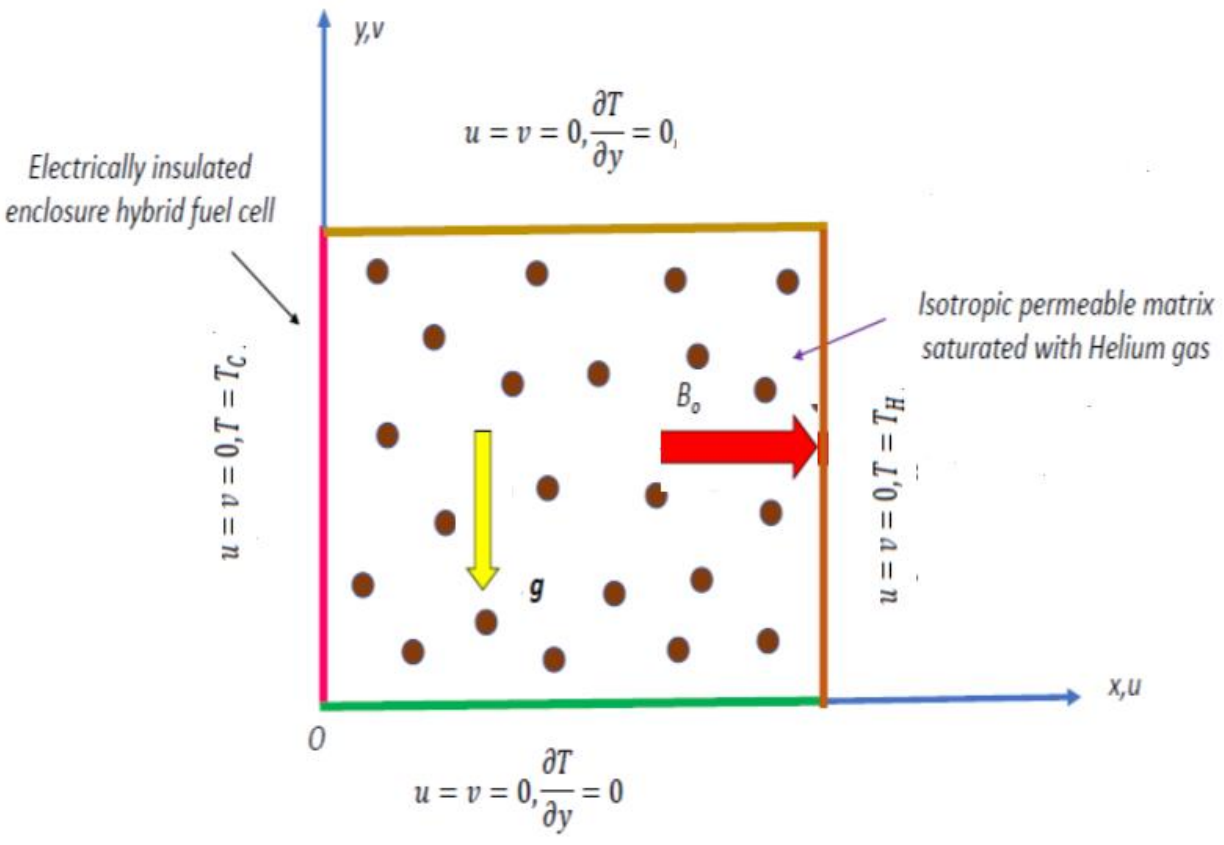

Fig 1: Physical model for hybrid electromagnetic Helium porous medium fuel cell enclosure

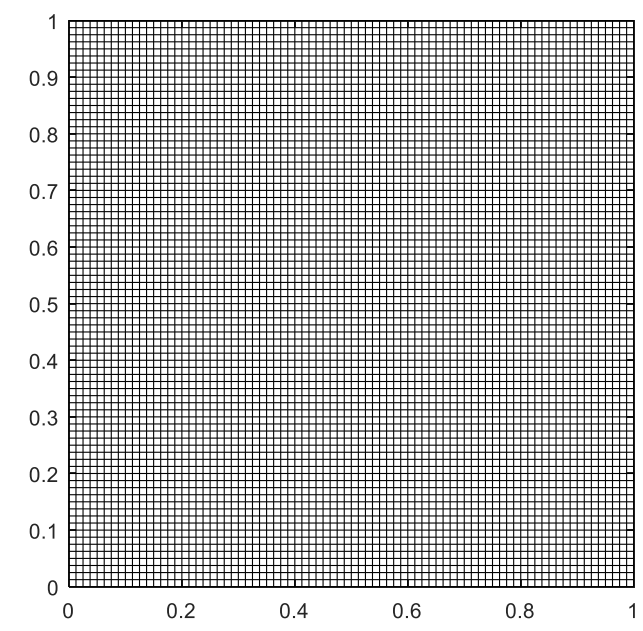

Figure 2: Uniform finite difference mesh for square enclosure (hybrid fuel cell geometry) 

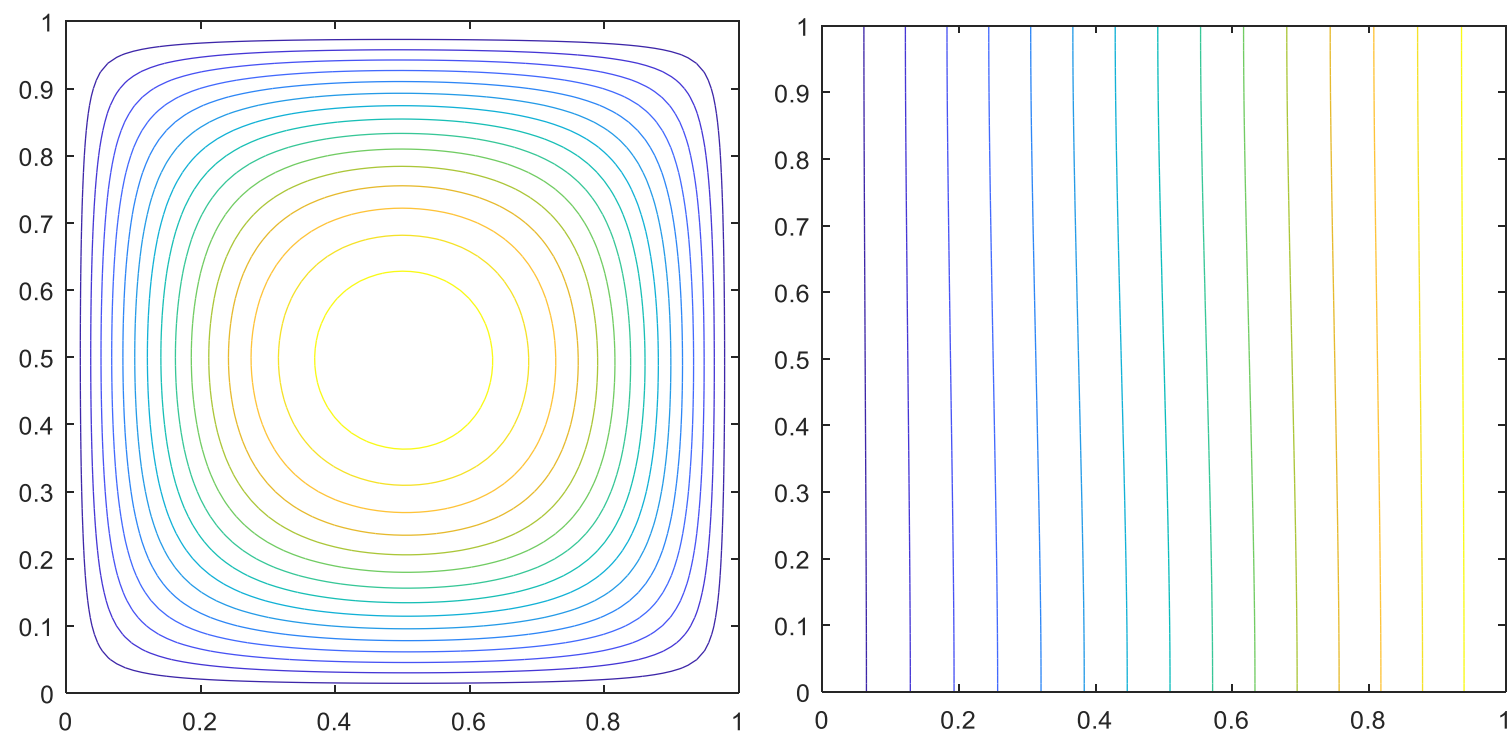

Fig. 3 a) Streamlines and b) Isotherms for $\mathrm{Gr}=2 X 10^{4}, \mathrm{Pr}=0.71 ; \mathrm{Ha}=5 ; \mathrm{Da}=0.0001 ; \Gamma=0$
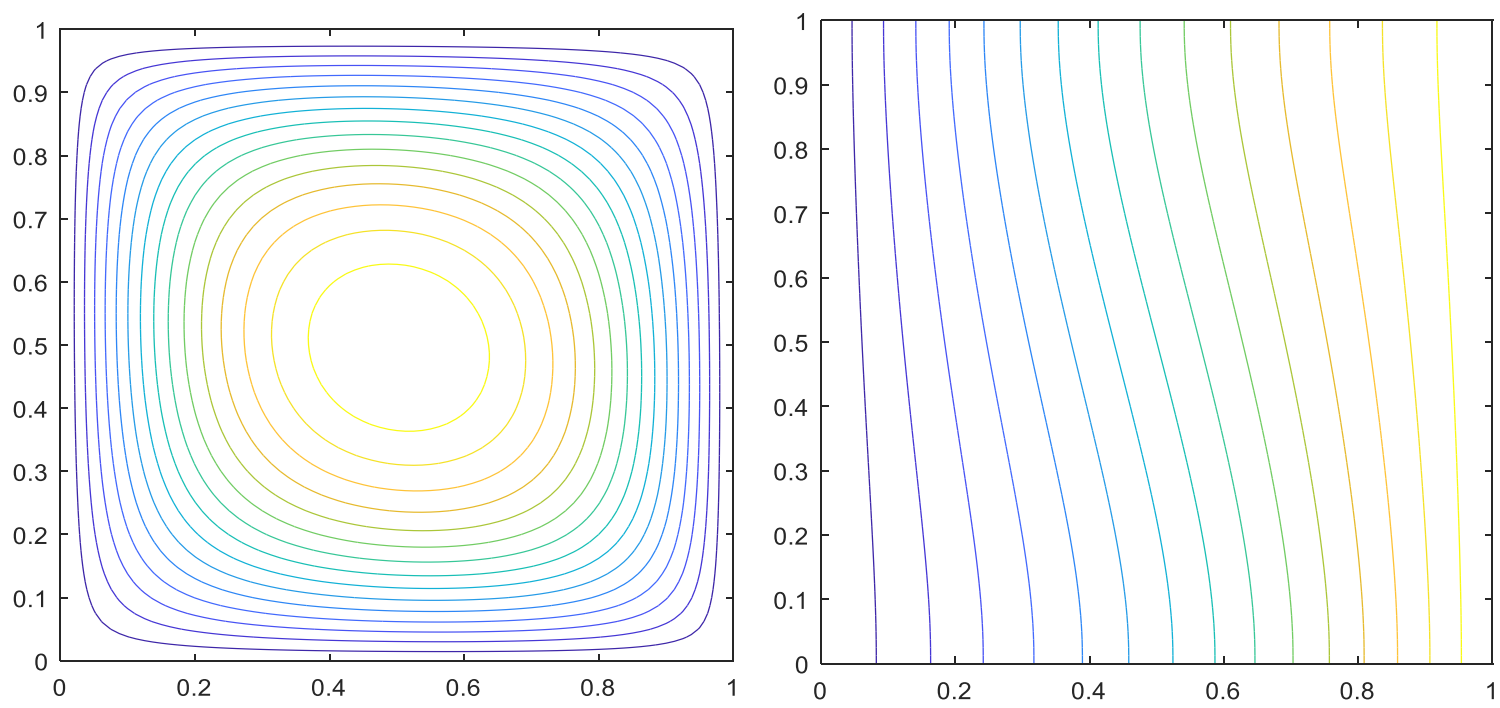

Fig. 4 a) Streamlines and b) Isotherms for $G r=2 \times 10^{5}, \mathrm{Pr}=0.71 ; \mathrm{Ha}=5 ; \mathrm{Da}=0.0001 ; \Gamma=0$ 



Fig. 5 a) Streamlines and b) Isotherms for $G r=2 X 10^{6}, P r=0.71, H a=5, D a=0.0001, \Gamma=0$
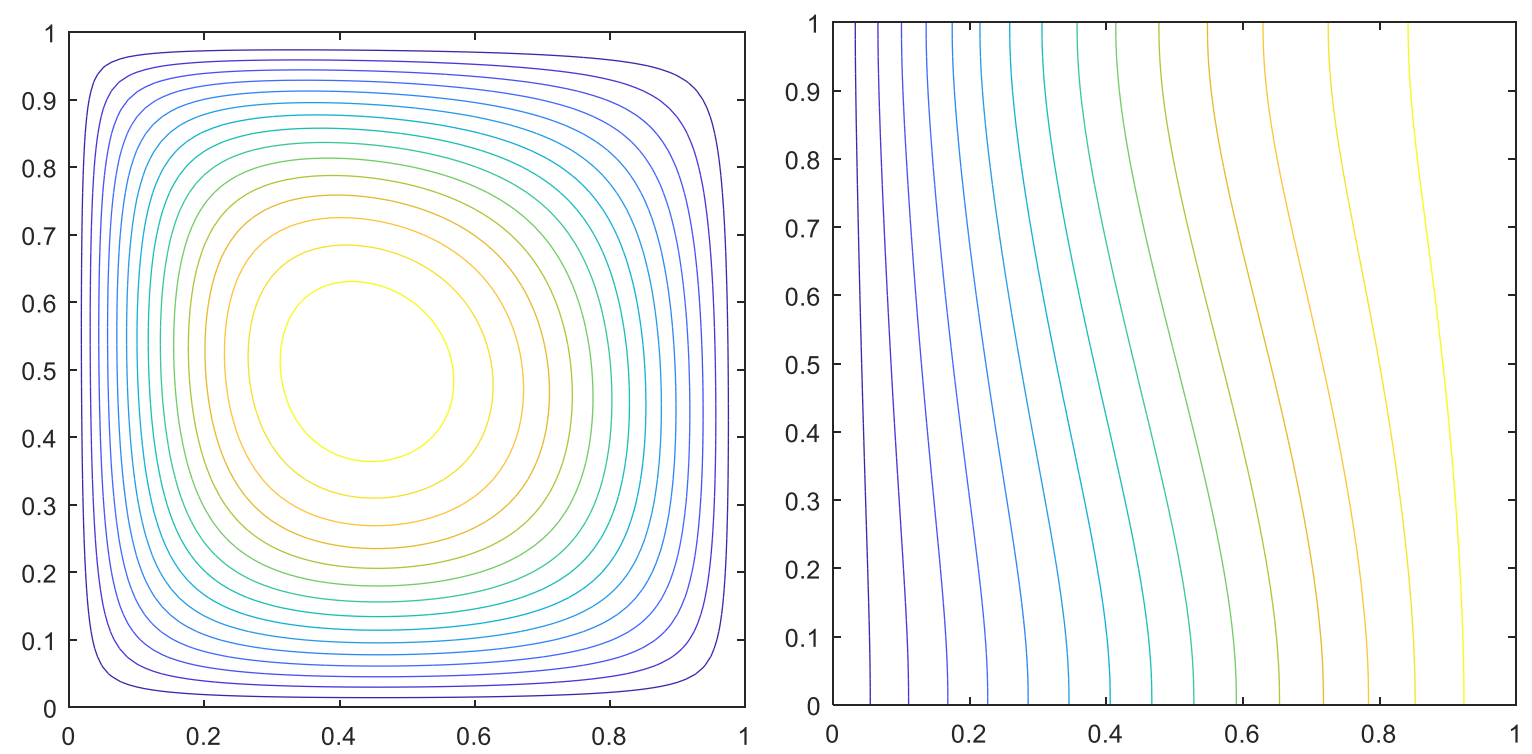

Fig 6 a) Streamlines and b) Isotherms for $G r=2 X 10^{5}, \operatorname{Pr}=0.71, \mathrm{Ha}=5, \mathrm{Da}=0.0001, \Gamma=1$ 

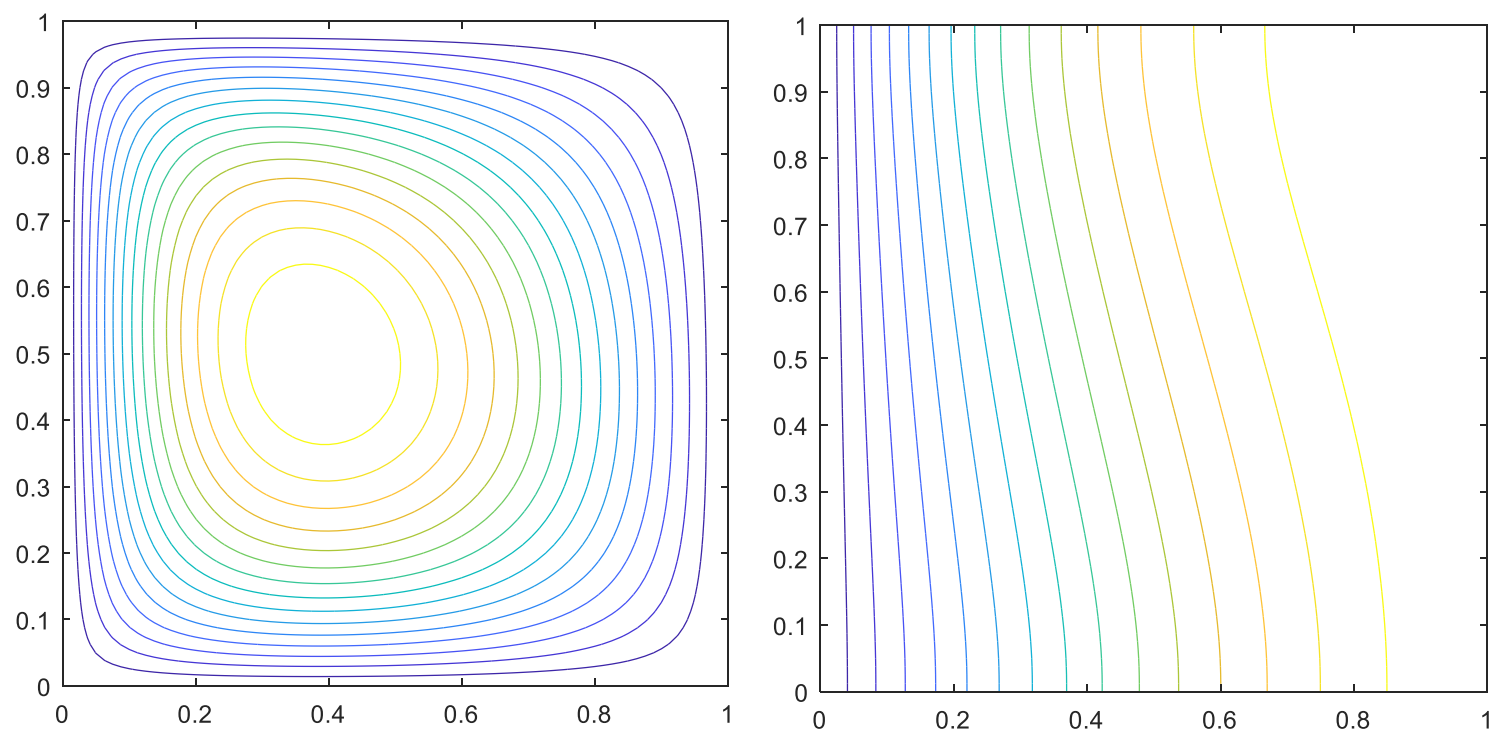

Fig. 7a) Streamlines and b) Isotherms for $G r=2 X 10^{5}, \mathrm{Pr}=0.71, \mathrm{Ha}=5, \mathrm{Da}=0.0001$ and $\Gamma=2$
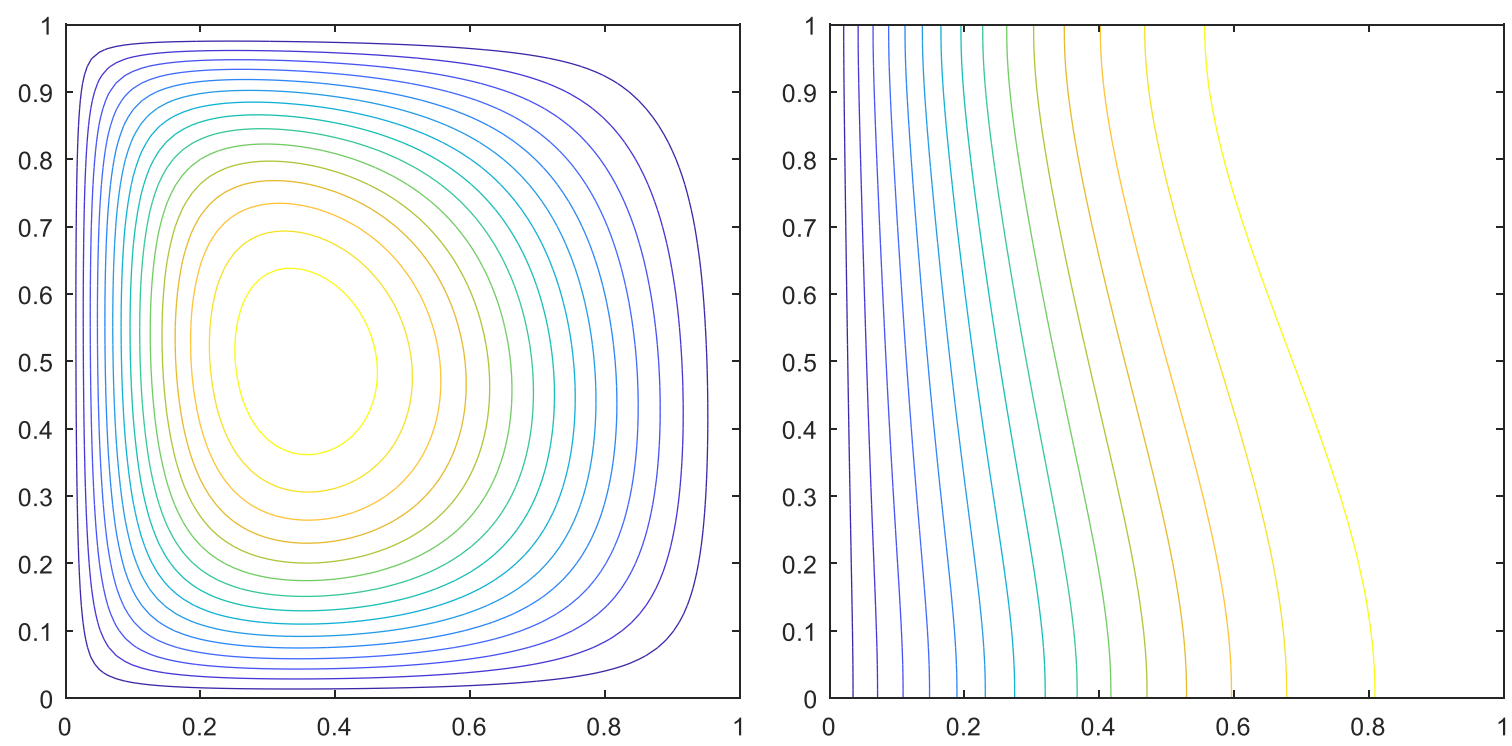

Fig. 8 a) Streamlines and b) Isotherms for $G r=2 X 10^{5}, \mathrm{Pr}=0.71, \mathrm{Ha}=5, \mathrm{Da}=0.0001$ and $\Gamma=3$ 

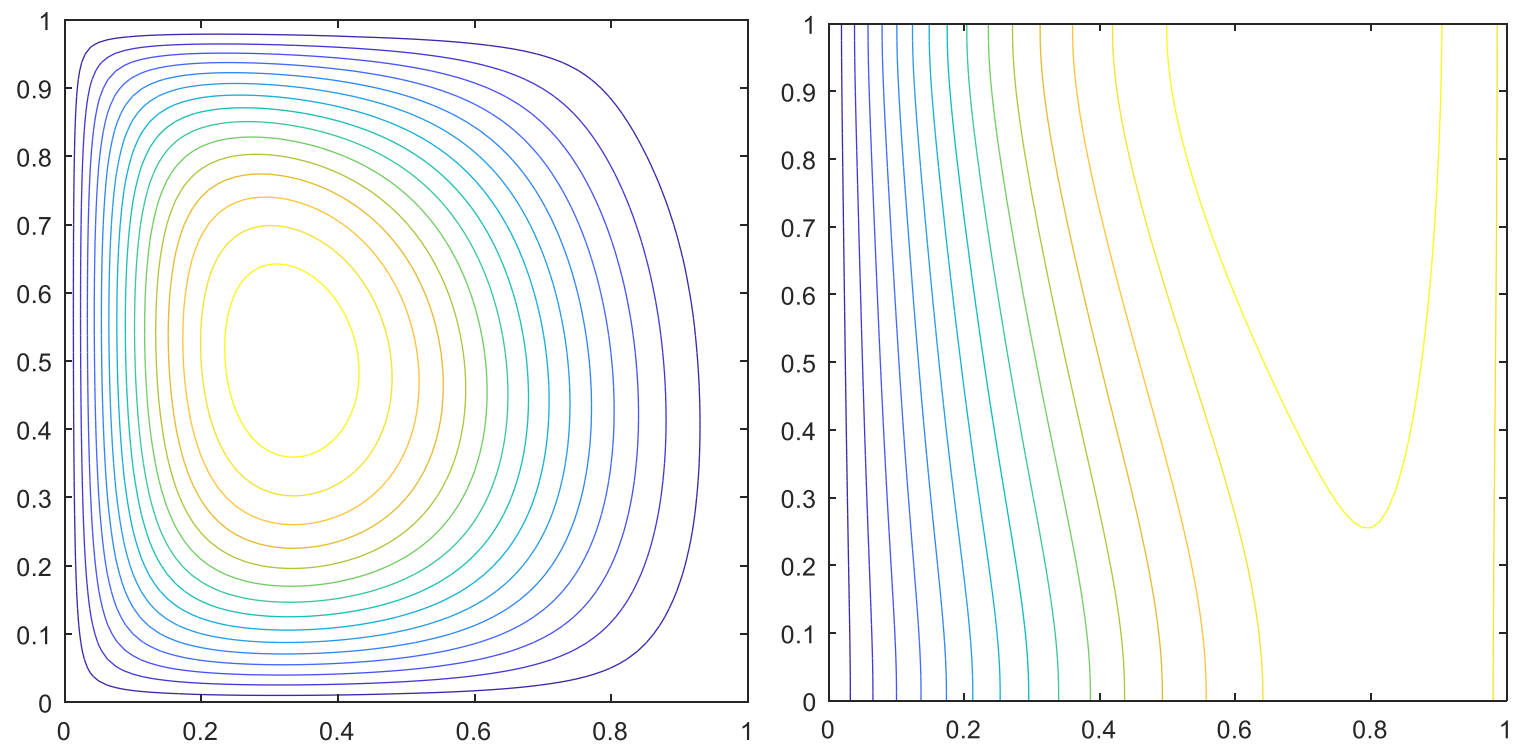

Fig. 9 a) Streamlines and b) Isotherms for $\mathrm{Gr}==2 X 10^{5} ; \mathrm{Pr}=0.71 ; \mathrm{Ha}=5 ; \mathrm{Da}=0.0001 ; \Gamma=4$
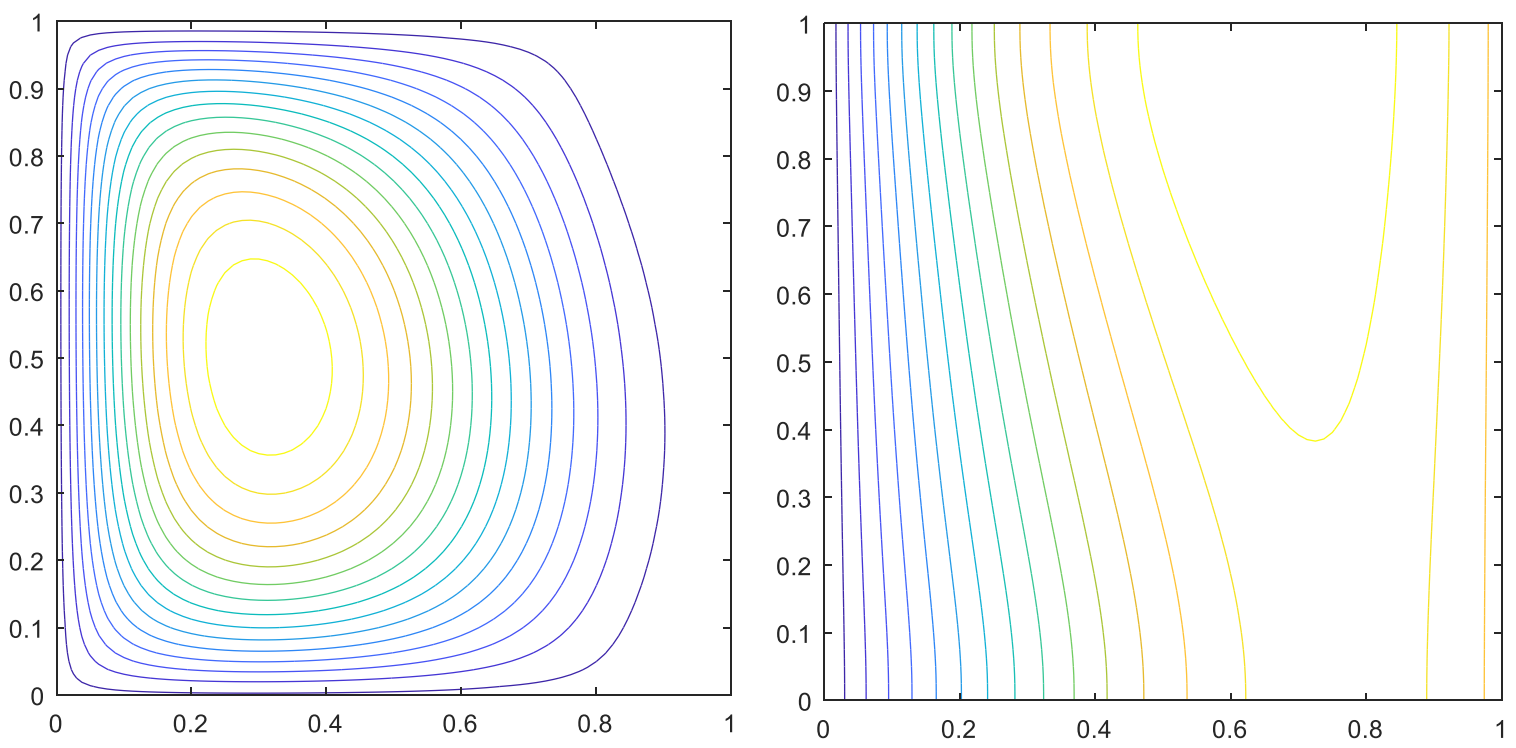

Fig. 10a) Streamlines and b) Isotherms for $G r=2 X 10^{5} ; \mathrm{Pr}=0.71 ; \mathrm{Ha}=5 ; \mathrm{Da}=0.0001 ; \Gamma=5$ 

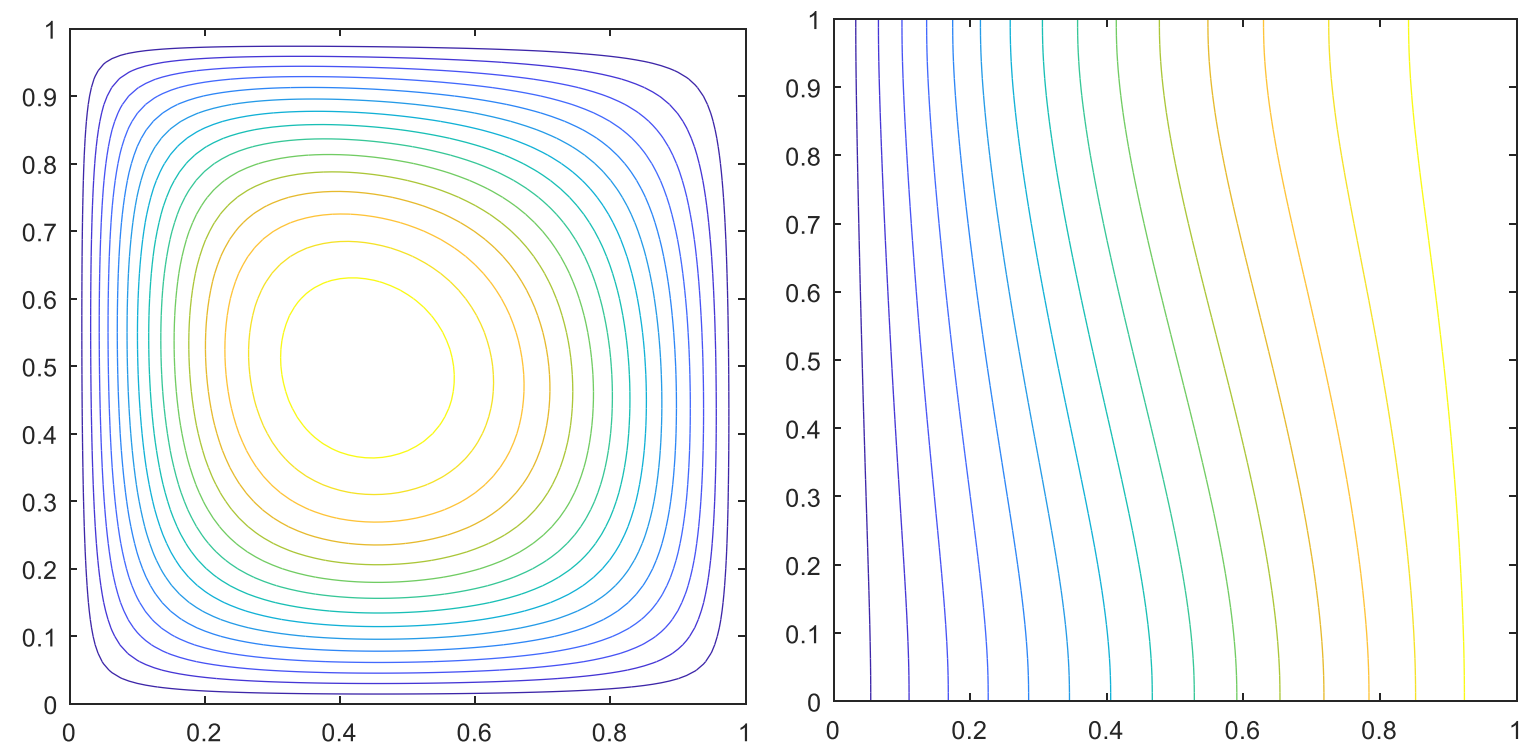

Fig 11a) Streamlines and b) Isotherms for $G r=2 X 10^{5} ; \mathrm{Pr}=0.71 ; \mathrm{Ha}=5 ; \Gamma=1 ; \mathrm{Da}=0.0001$.
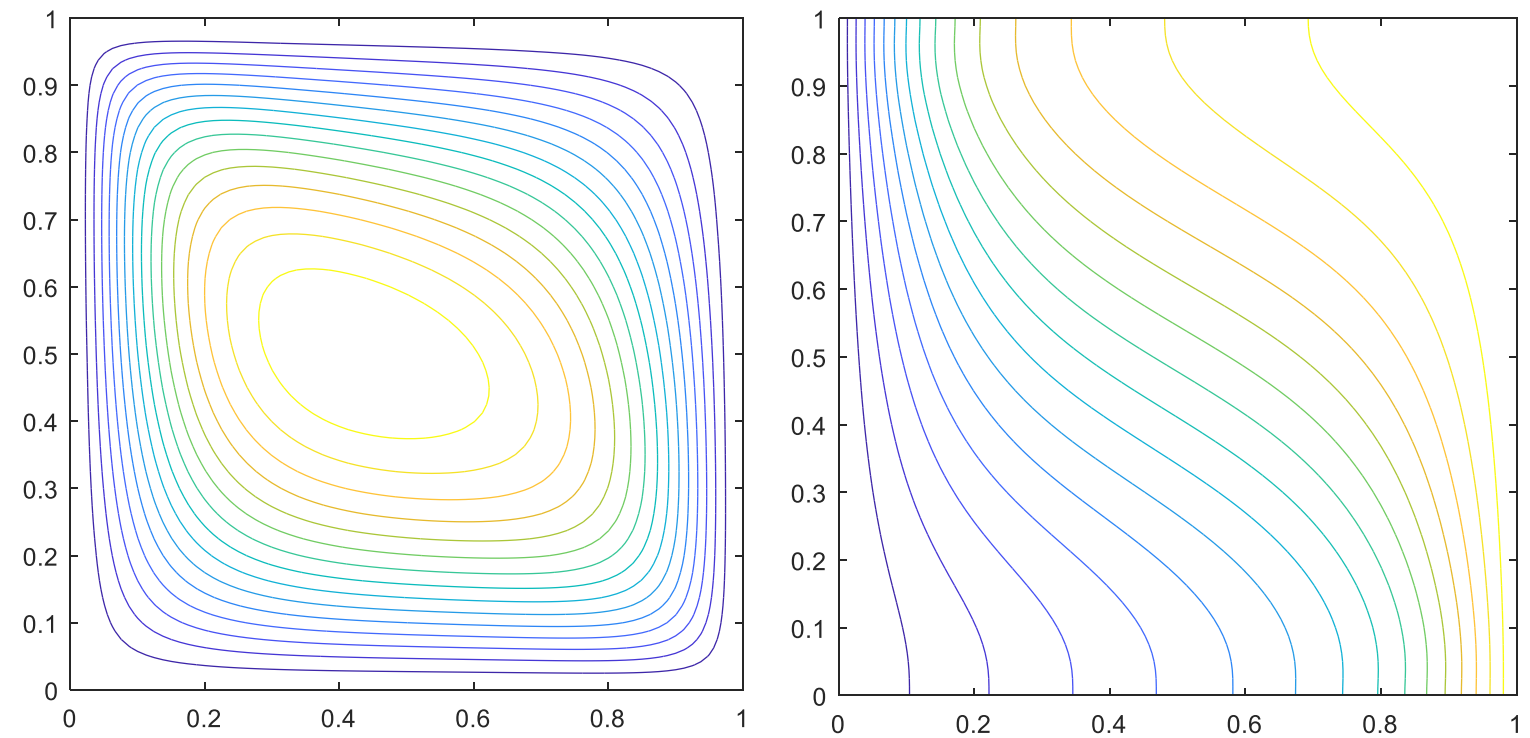

Fig 12 a) Streamlines and b) Isotherms for $G r=2 X 10^{5}, \mathrm{Pr}=0.71, \mathrm{Ha}=5, \Gamma=1, \mathrm{Da}=0.001$ 



Fig 13a) Streamlines and b) Isotherms for $G r=2 X 10^{5}, \operatorname{Pr}=0.71, \mathrm{Ha}=5, \Gamma=1, \mathrm{Da}=0.01$
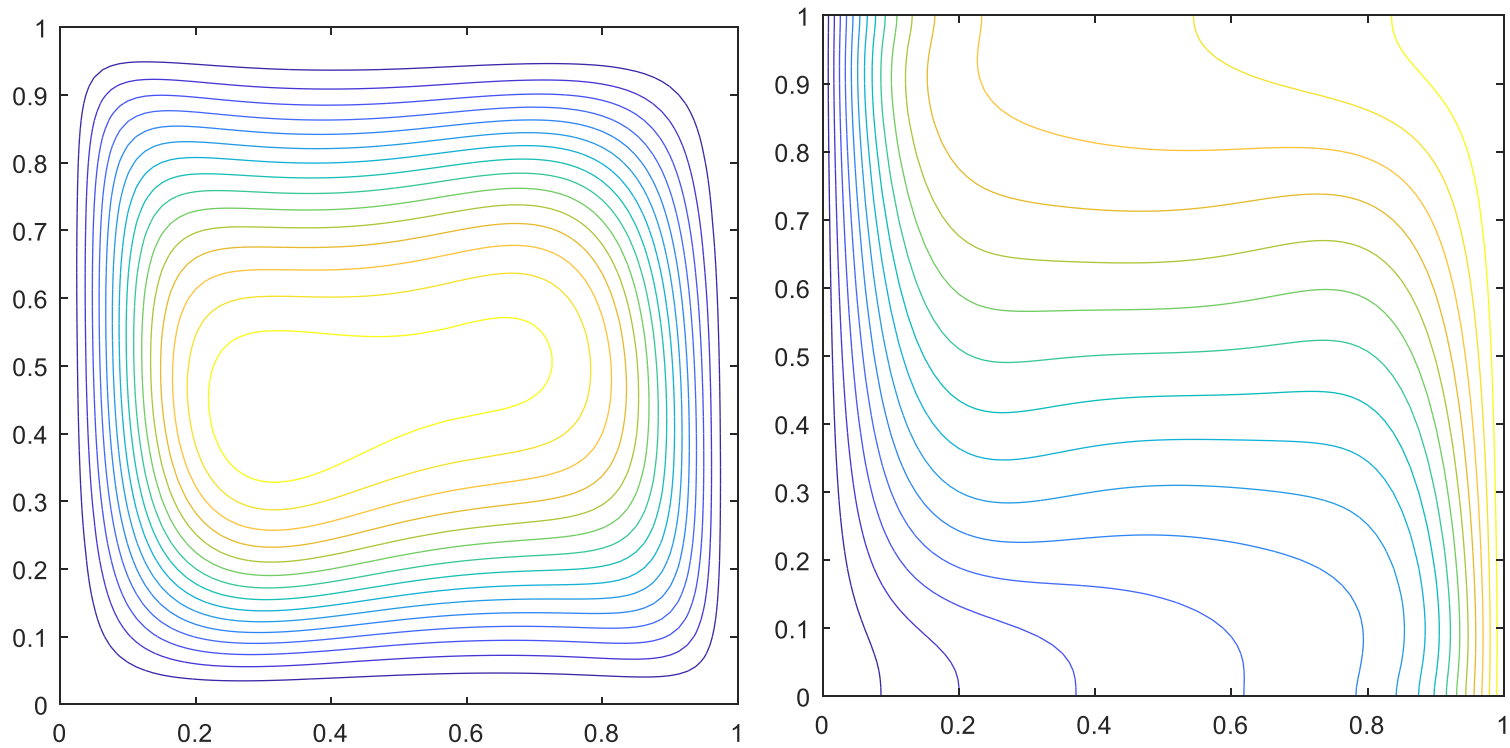

Fig 14a) Streamlines and b) Isotherms for $G r=2 \times 10^{5}, \operatorname{Pr}=0.71, \mathrm{Ha}=5, \Gamma=1, \mathrm{Da}=0.1$ 

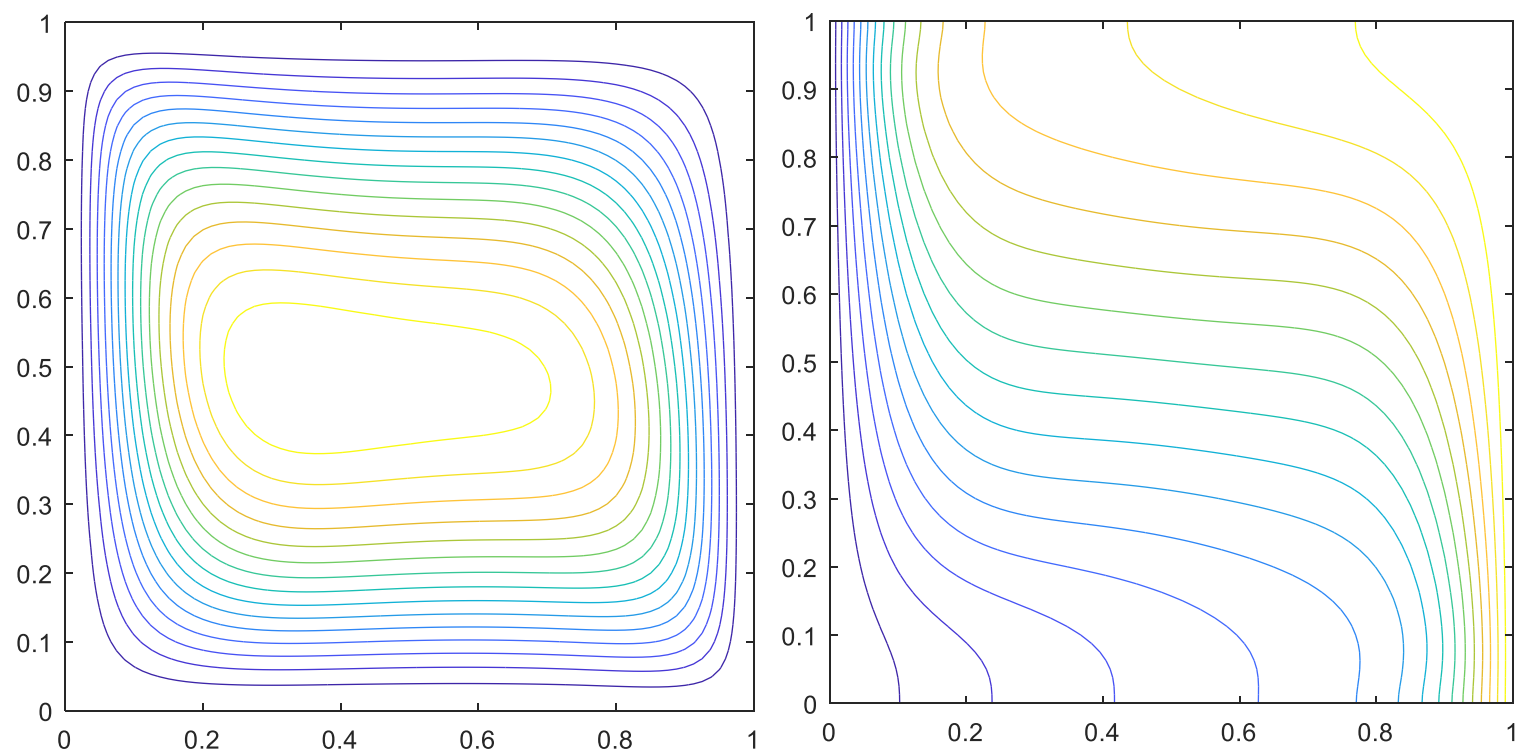

Fig 15a) Streamlines and b) Isotherms for $G r=2 X 10^{5}, \operatorname{Pr}=0.71, \Gamma=1, D a=0.01, H a=5$
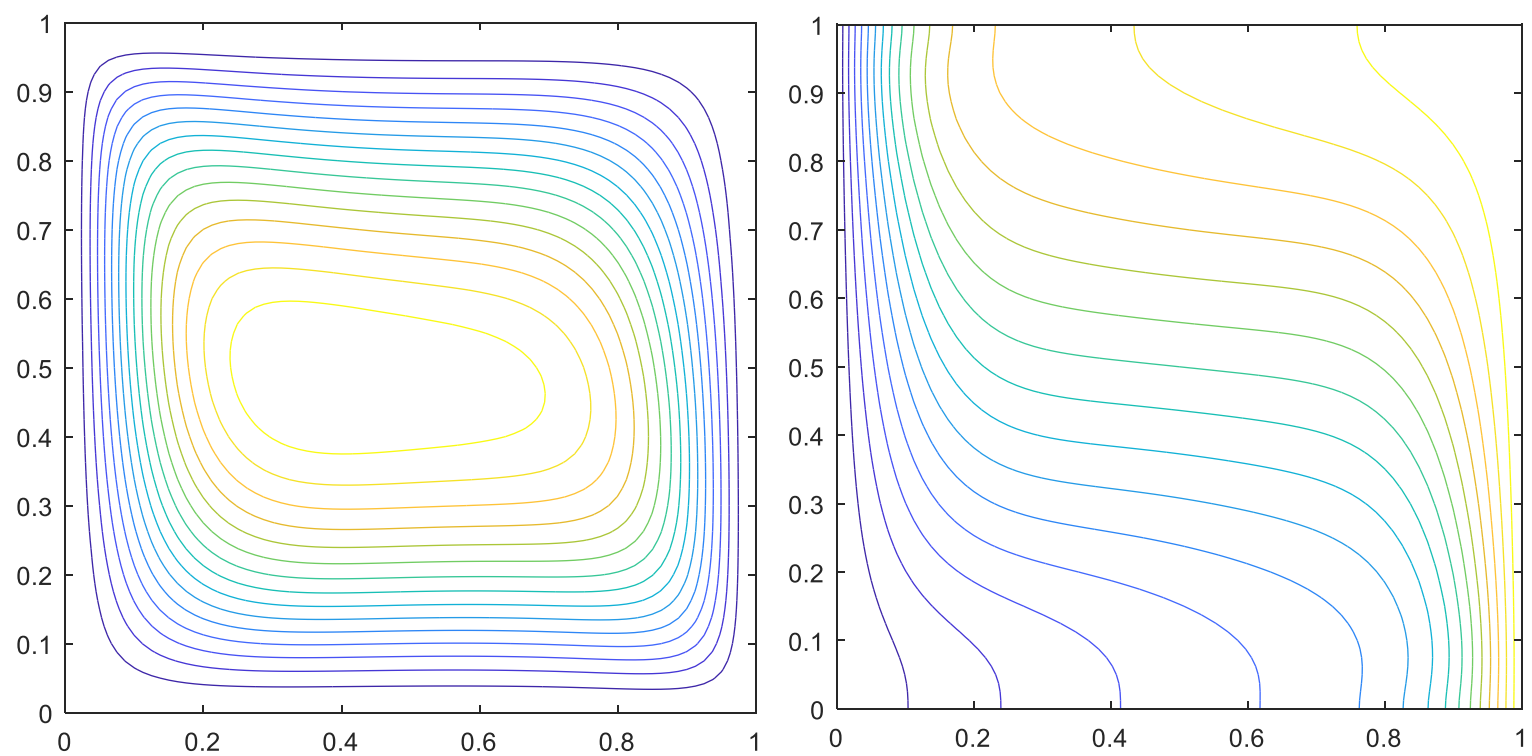

Fig 16 a) Streamlines and b) Isotherms for $G r=2 X 10^{5}, \operatorname{Pr}=0.71, \Gamma=1, \mathrm{Da}=0.01, \mathrm{Ha}=10$ 

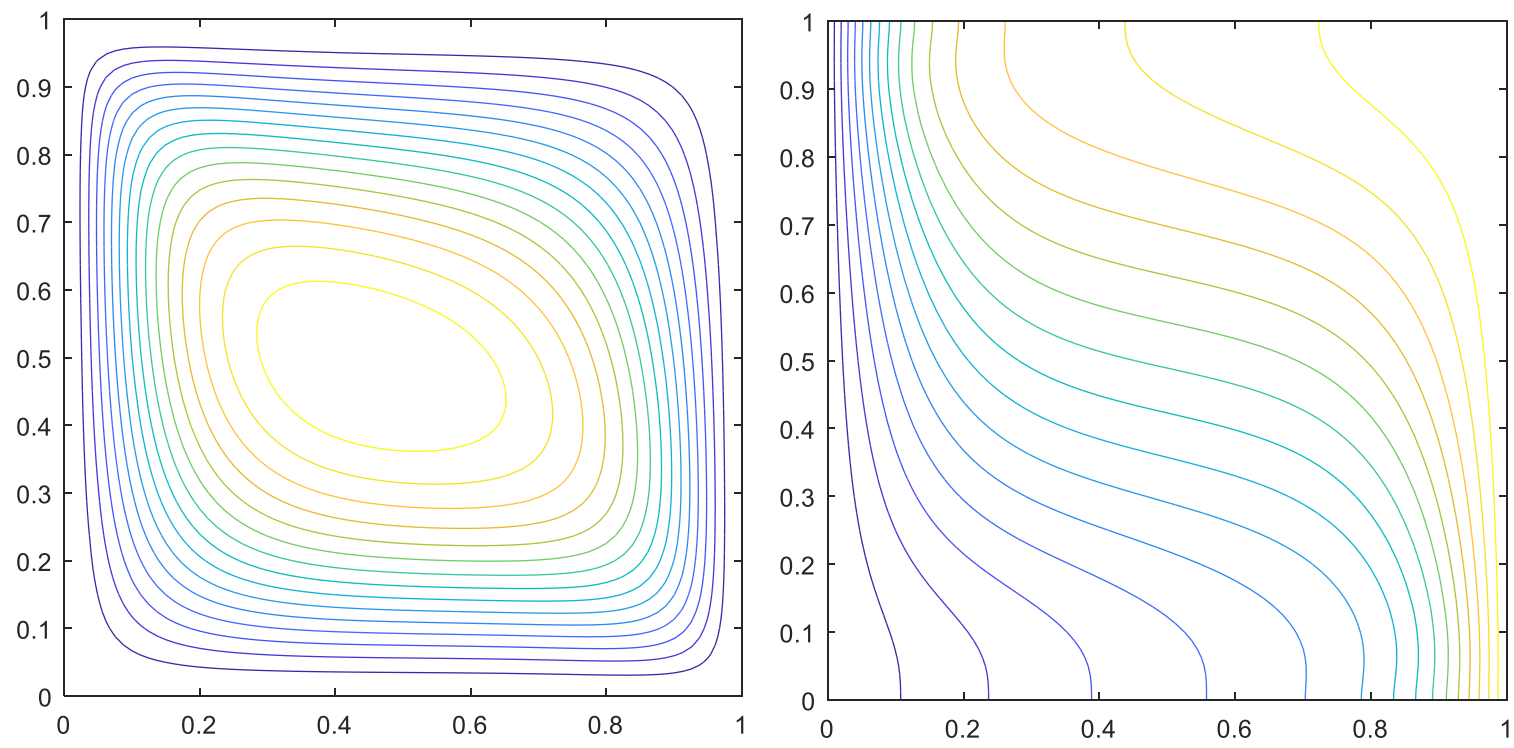

Fig 17a) Streamlines and b) Isotherms for $G r=2 X 10^{5}, \operatorname{Pr}=0.71, \Gamma=1, \mathrm{Da}=0.01, \mathrm{Ha}=25$
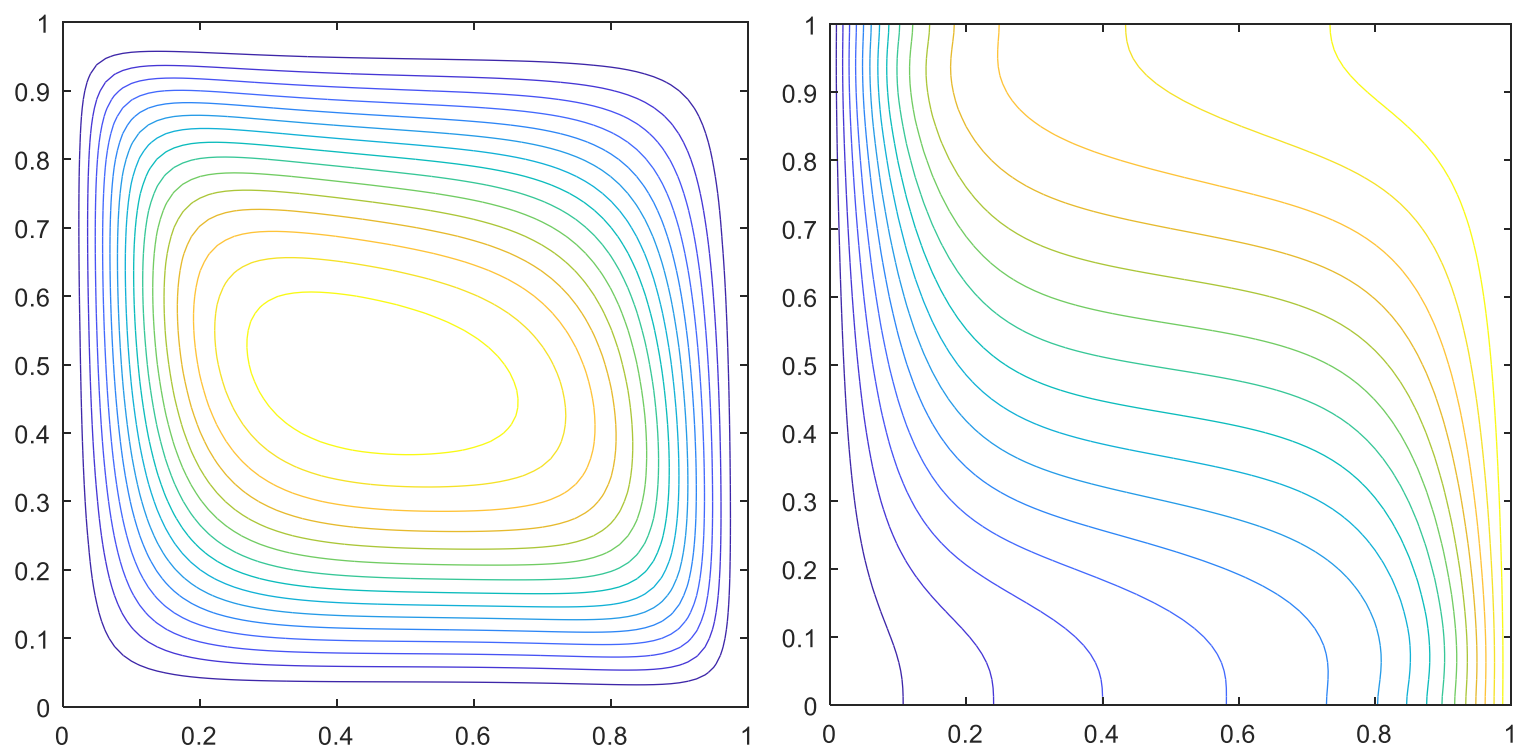

Fig 18a) Streamlines and b) Isotherms for $G r=2 X 10^{5}, \operatorname{Pr}=0.71, \Gamma=1, D a=0.01, H a=30$ 

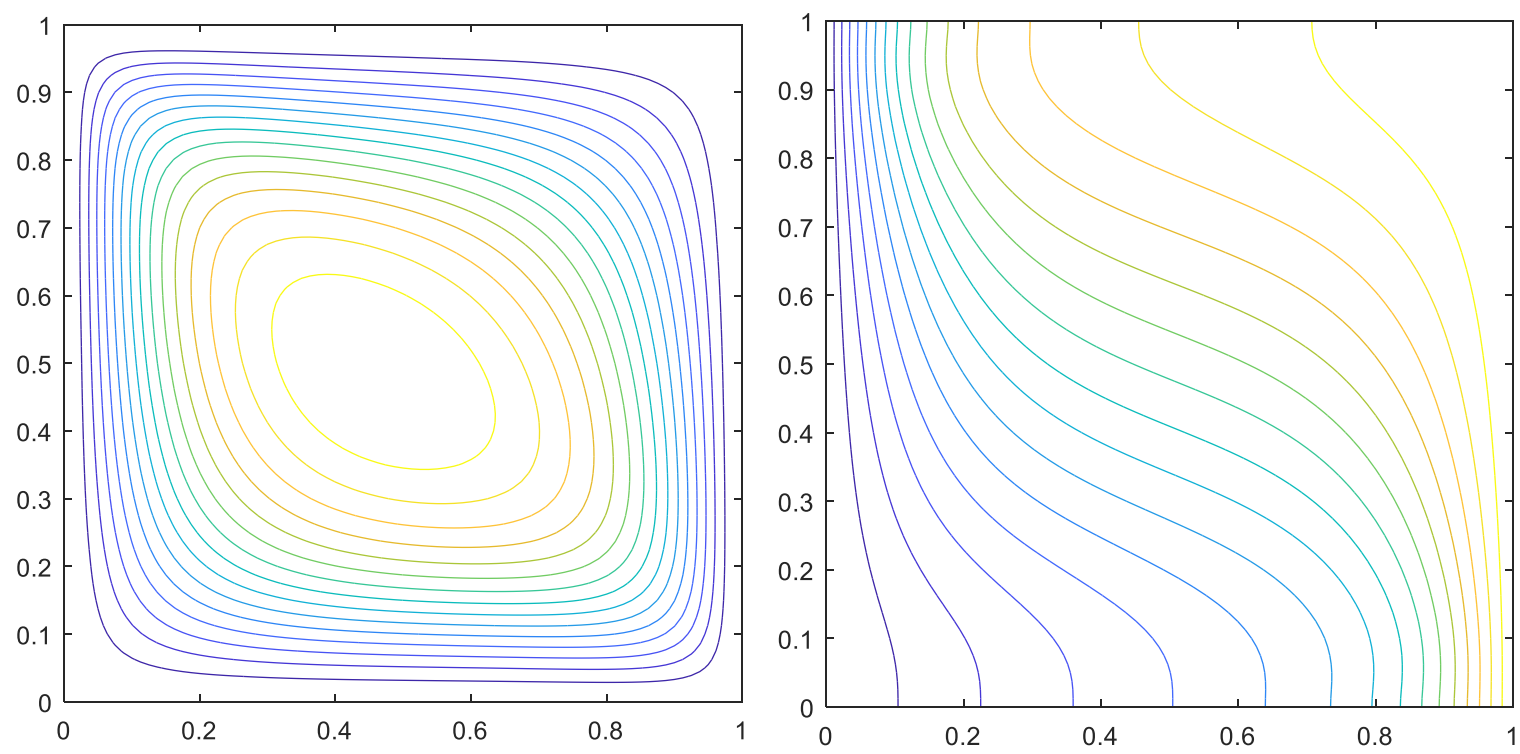

Fig 19a) Streamlines and b) Isotherms for $G r=2 X 10^{5}, \operatorname{Pr}=0.71, \Gamma=1, D a=0.01, H a=35$
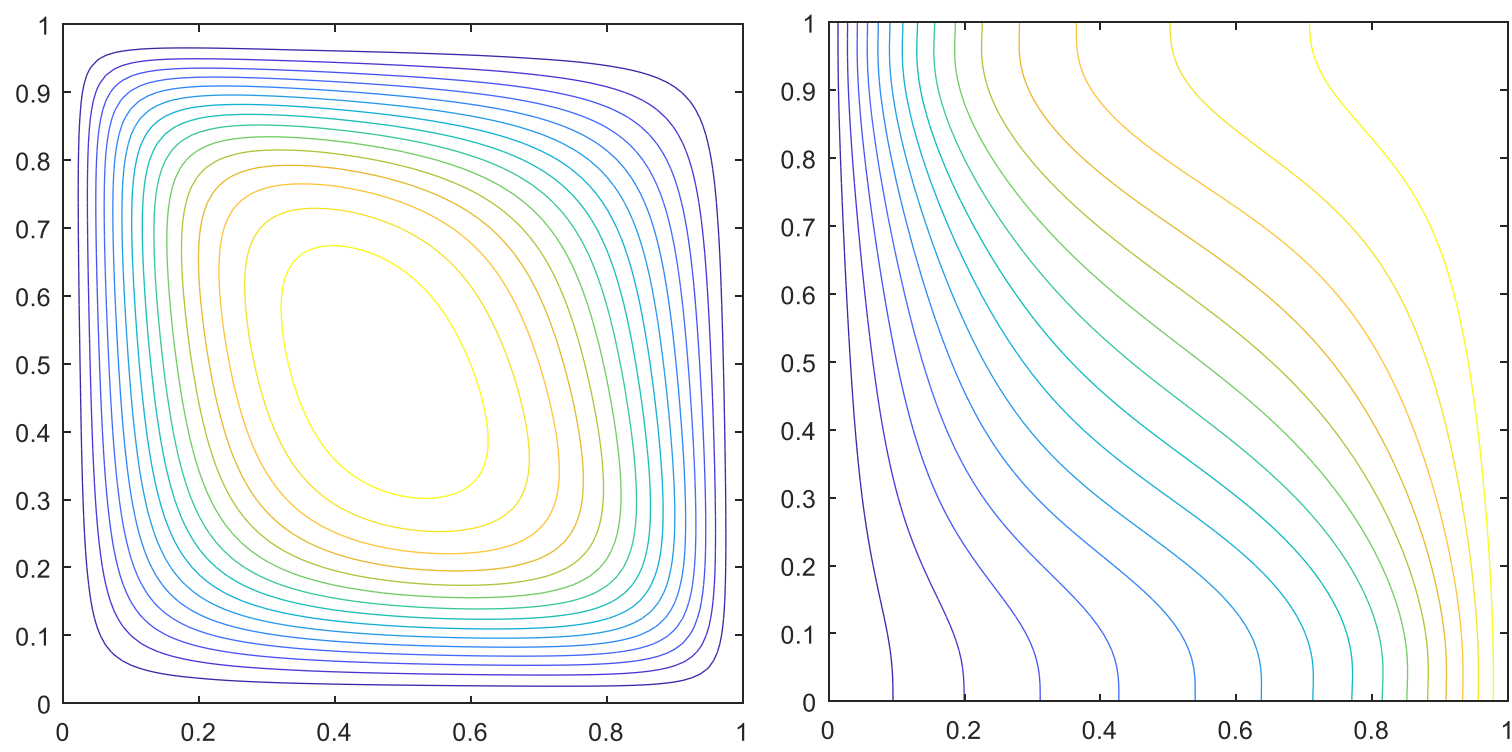

Fig 20a) Streamlines and b) Isotherms for $G r=2 X 10^{5}, \operatorname{Pr}=0.71, \Gamma=1, \mathrm{Da}=0.01, \mathrm{Ha}=50$ 


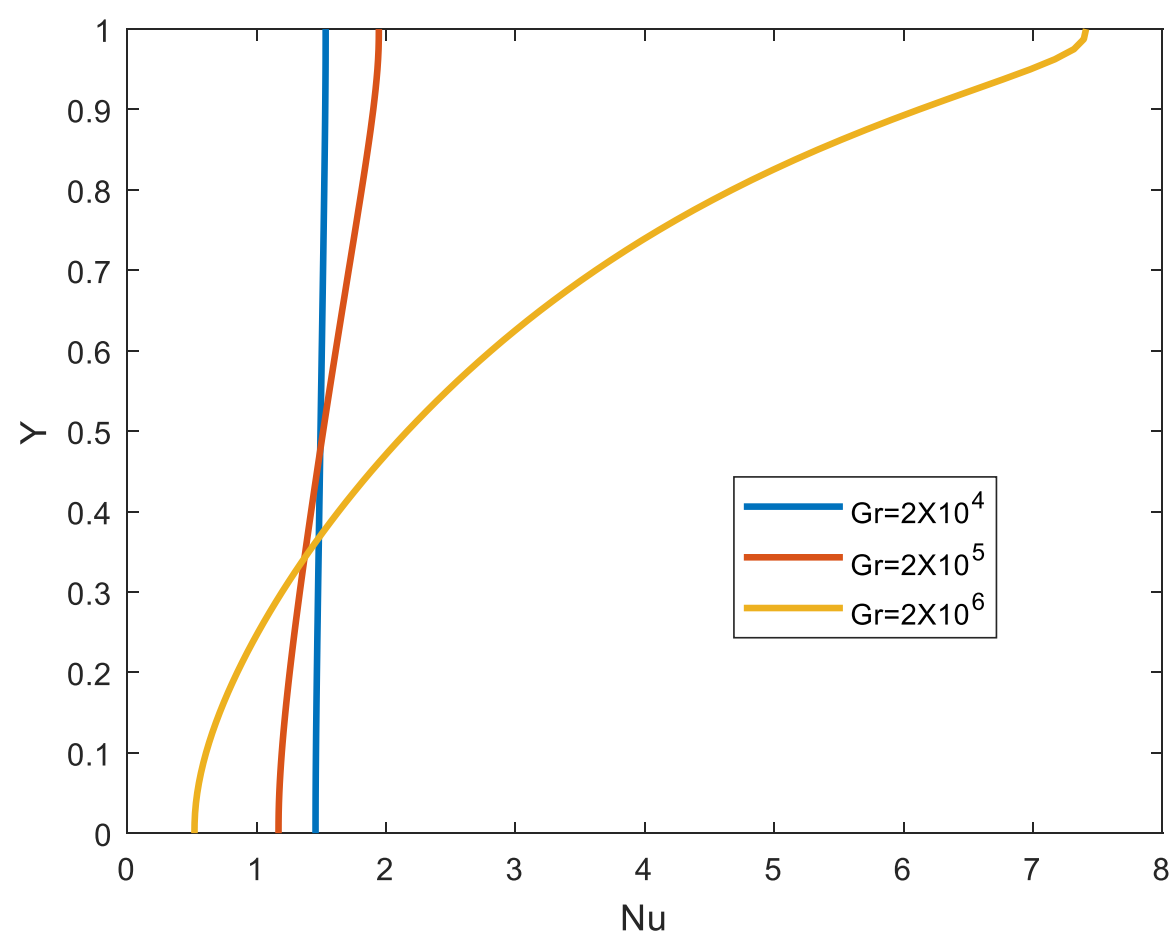

a) Left wall

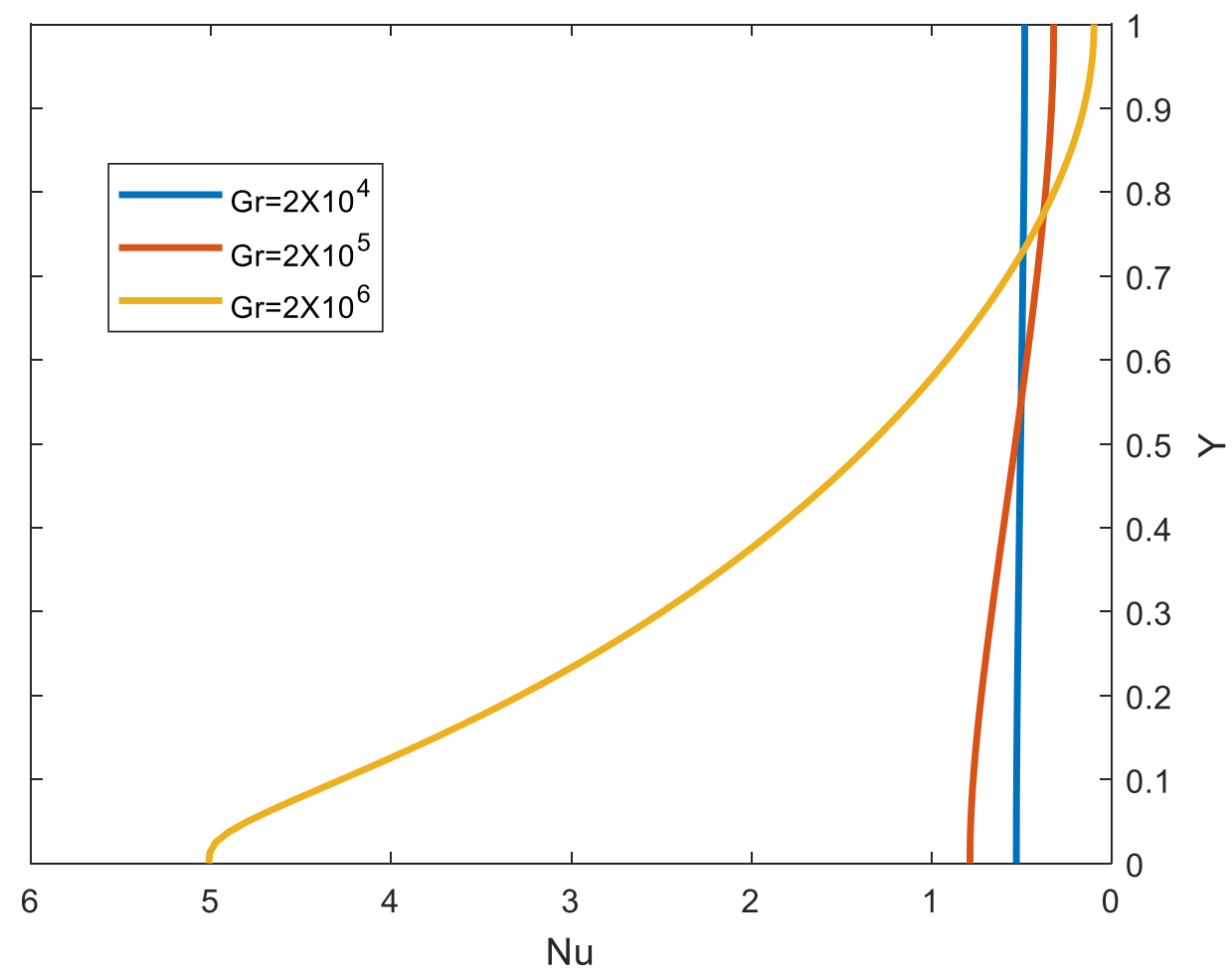

b) Right wall

Fig. 21 Local Nusselt number for a) left cold wall, b) right hot wall with $\operatorname{Pr}=0.71, \mathrm{Ha}=5$, Da $=0.0001, \Gamma=1$ for different Grashof (thermal buoyancy) numbers $(G r)$ 


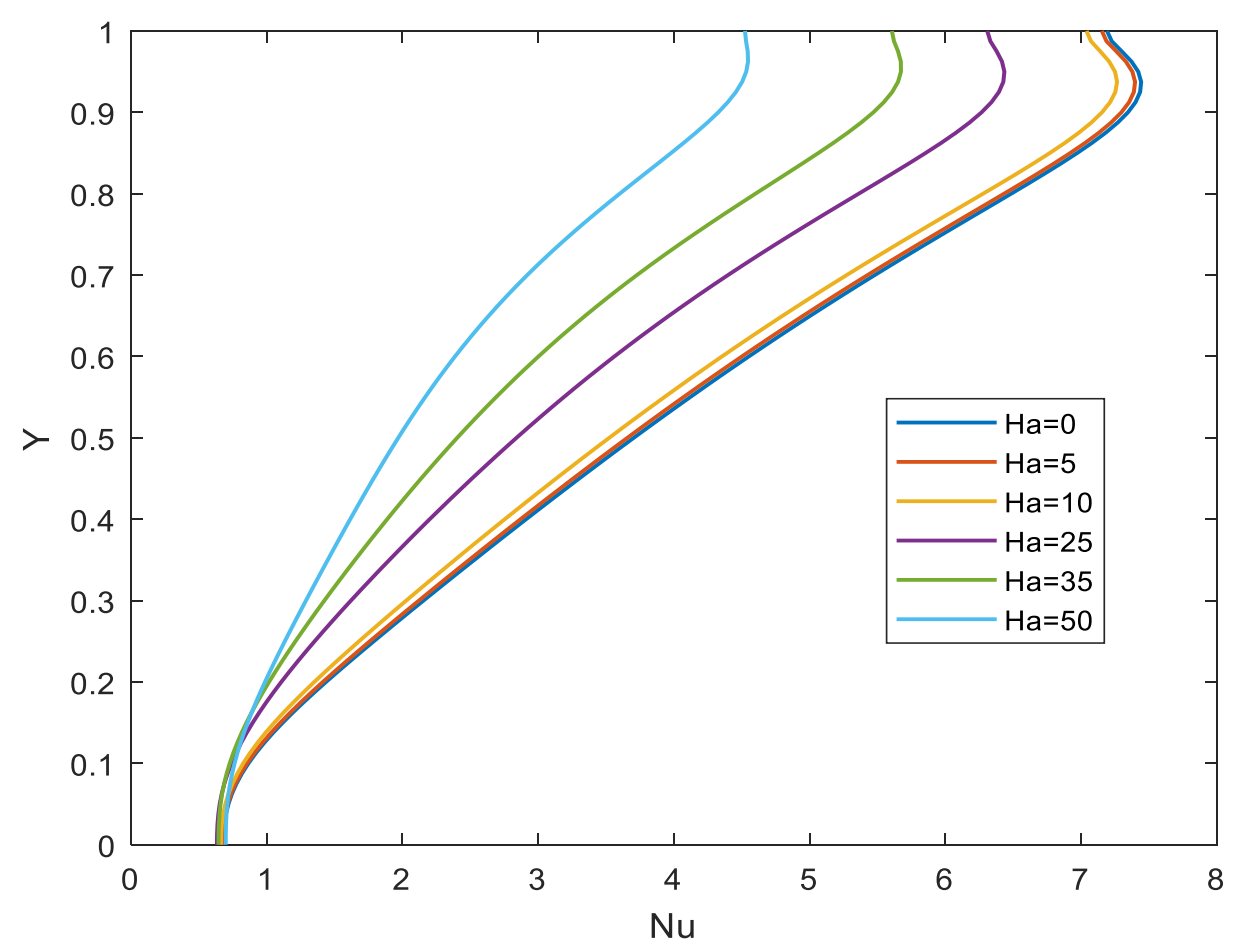

a) Left wall

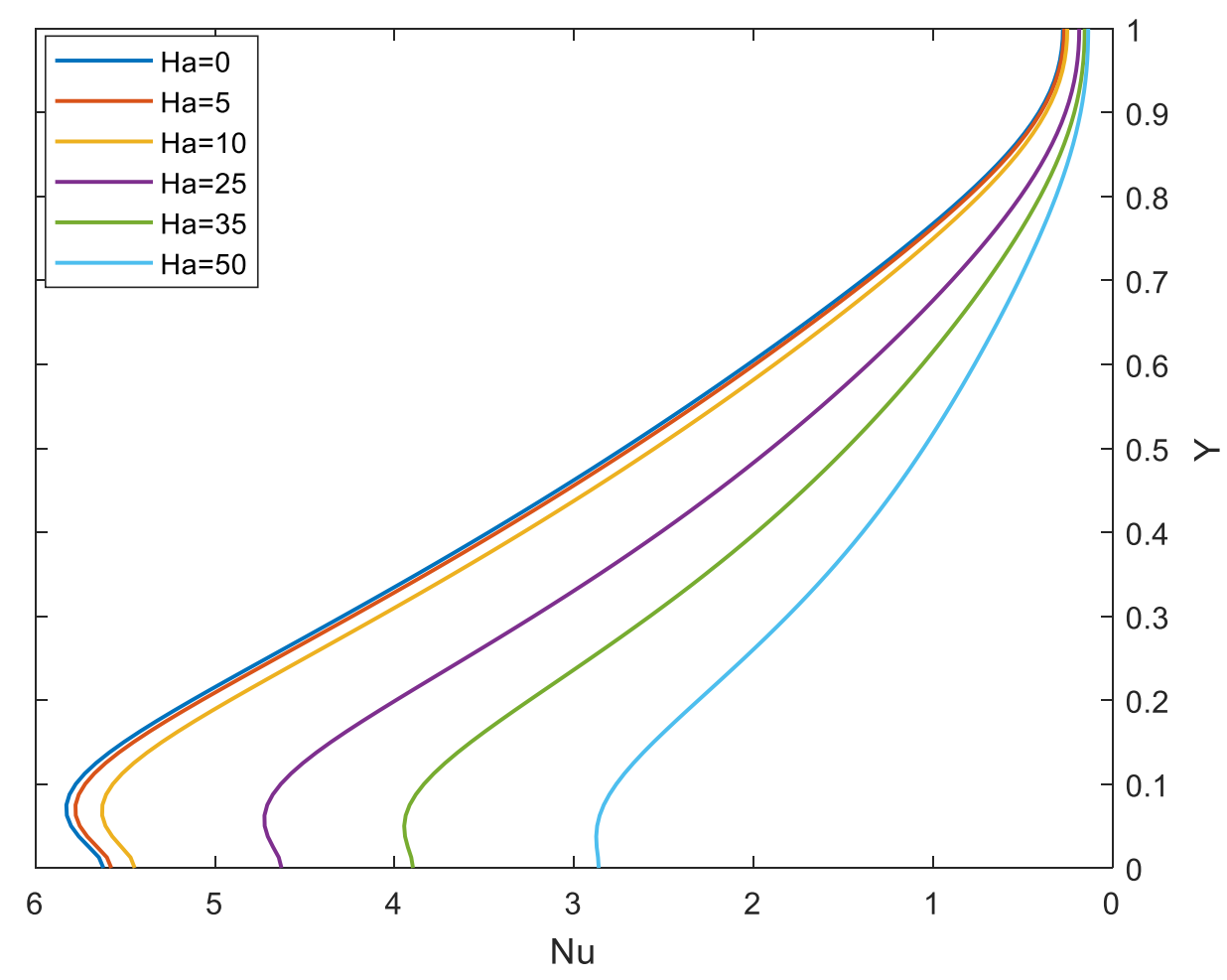

b) Right wall

Fig. 22 Local Nusselt number for a) left cold wall, b) right hot wall with $G r=2 \times 10^{5}, \operatorname{Pr}=$ 0.71, Da $=0.01, \Gamma=1$ for different Hartmann magnetic numbers $(\mathrm{Ha})$ 


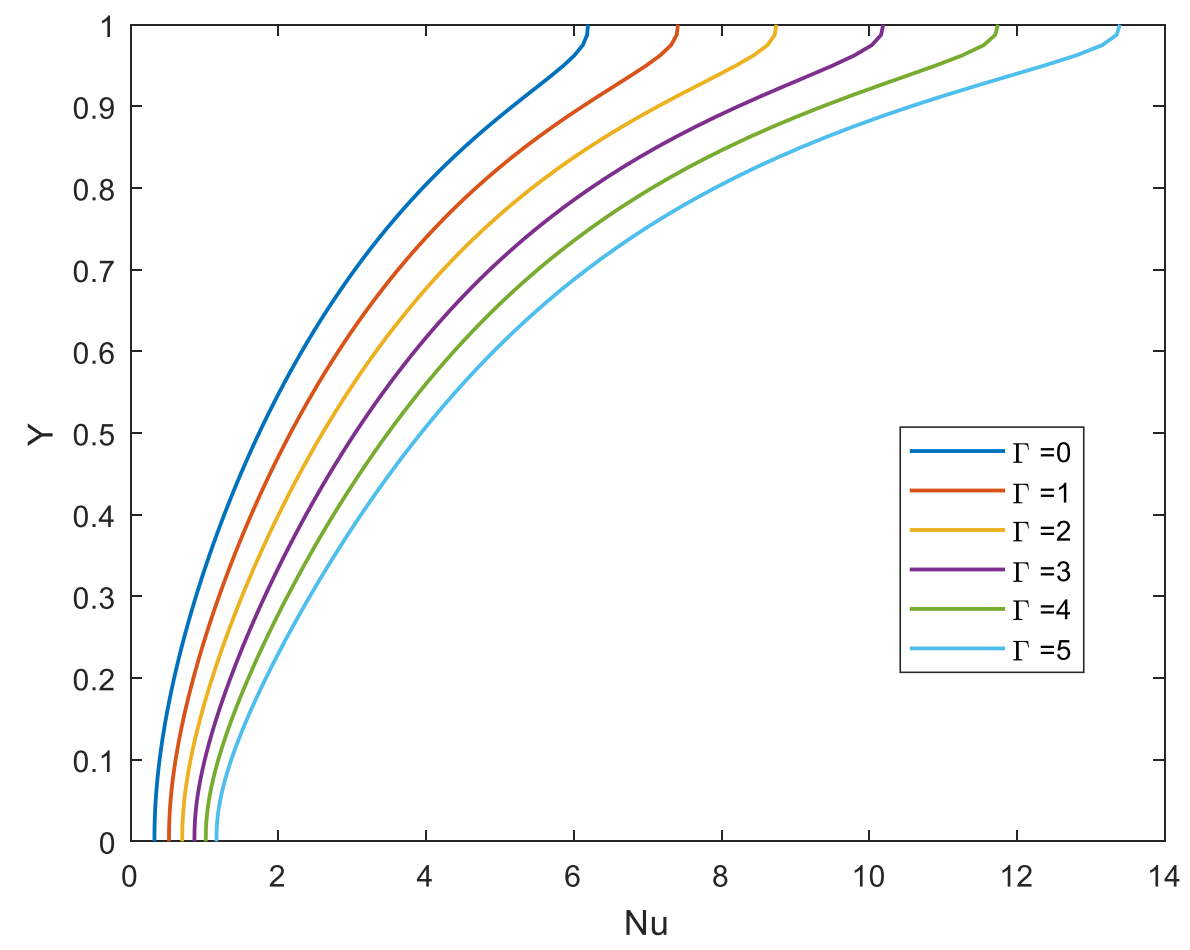

a) Left wall



b) Right wall

Fig. 23 Local Nusselt number for a) left cold wall, b) right hot wall with $\operatorname{Pr}=0.71, \mathrm{Ha}=5$, $D a=0.0001, G r=2 X 10^{5}$ for different internal heat source parameters $(\Gamma)$ 


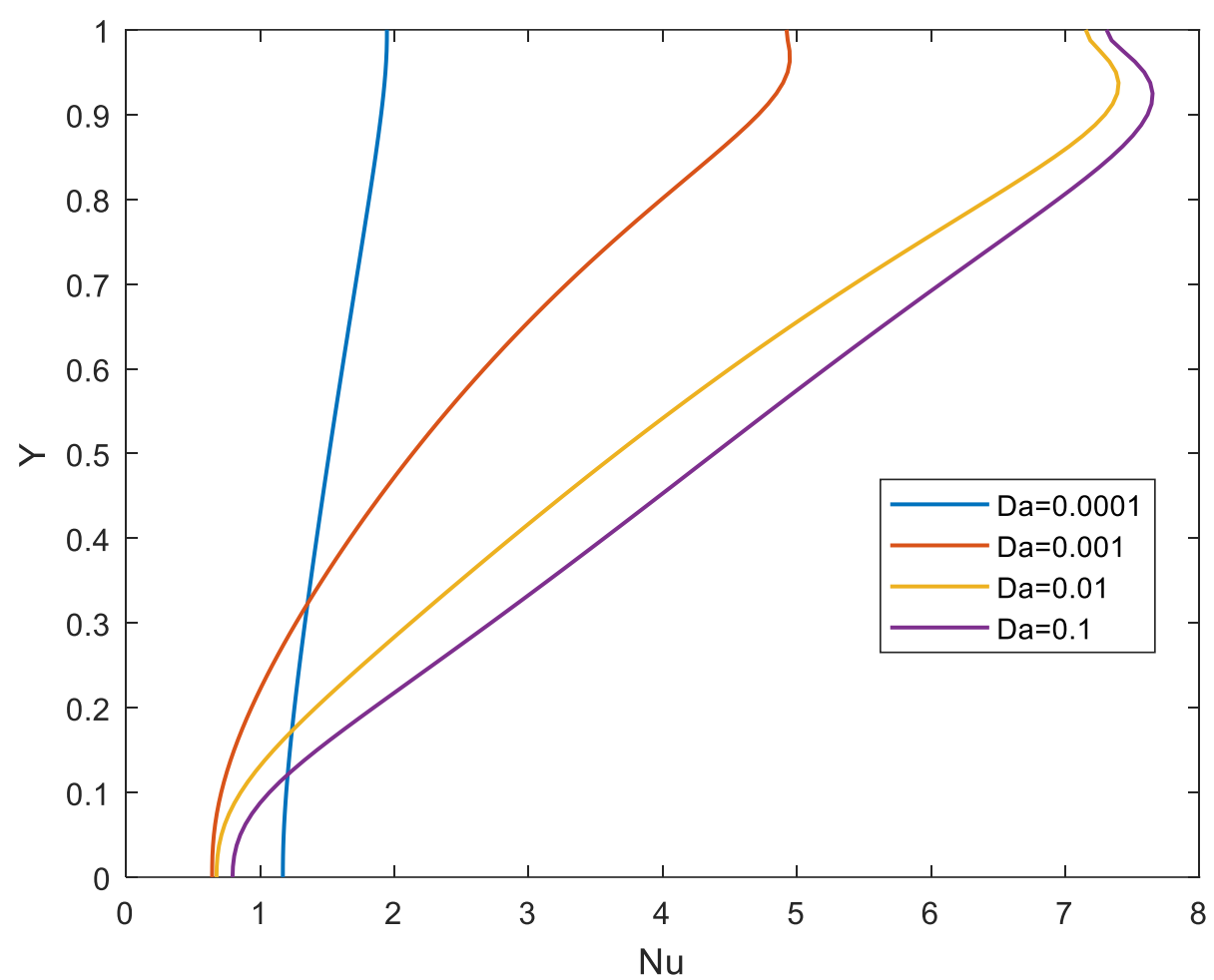

a) Left wall

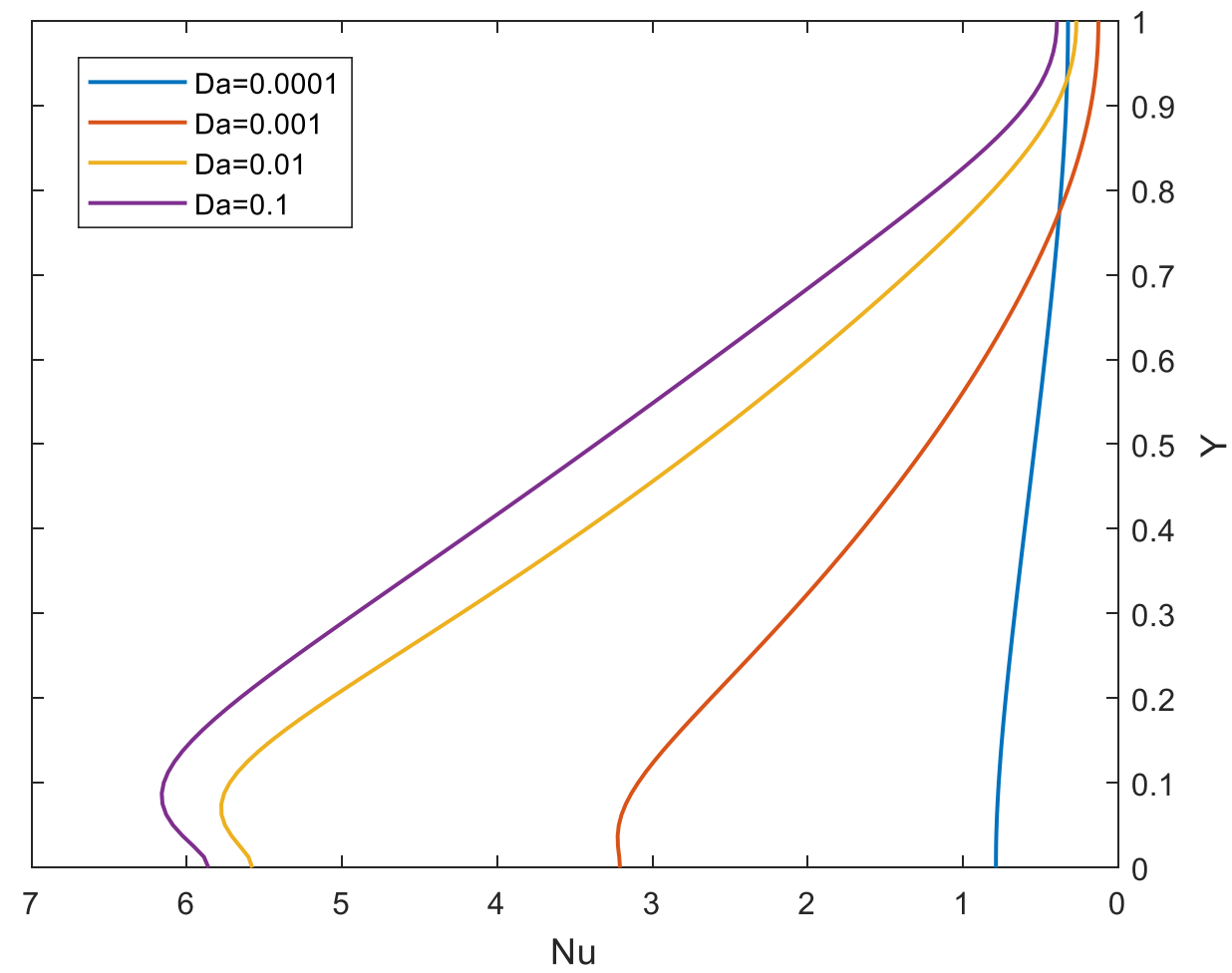

b) Right wall

Fig. 24 Local Nusselt number for a) left cold wall, b) right hot wall with $\operatorname{Pr}=0.71, \mathrm{Ha}=5$,

$$
\Gamma=1, G r=2 \times 10^{5} \text { for different Darcy numbers }(\mathrm{Da})
$$

